\title{
Standard model plethystics
}

\author{
Yan Xiao, ${ }^{1, *}$ Yang-Hui He, ${ }^{1,2,3, \dagger}$ and Cyril Matti ${ }^{1, *}$ \\ ${ }^{1}$ Department of Mathematics, City, University of London, \\ Northampton Square, London ECIV OHB, United Kingdom \\ ${ }^{2}$ Merton College, University of Oxford, Oxford OX1 4JD, United Kingdom \\ ${ }^{3}$ School of Physics, NanKai University, Tianjin 300071, People's Republic of China,
}

(Received 4 June 2019; published 3 October 2019)

\begin{abstract}
We study the vacuum geometry prescribed by the gauge invariant operators of the minimal supersymmetric standard model via the plethystic program. This is achieved by using several tricks to perform the highly computationally challenging Molien-Weyl integral, from which we extract the Hilbert series, encoding the invariants of the geometry at all degrees. The fully refined Hilbert series is presented as the explicit sum of 1422 rational functions. We found a good choice of weights to unrefine the Hilbert series into a rational function of a single variable, from which we can read off the dimension and the degree of the vacuum moduli space of the minimal supersymmetric standard model gauge invariants. All data in Mathematica format are also presented.
\end{abstract}

DOI: 10.1103/PhysRevD.100.076001

\section{INTRODUCTION AND SUMMARY}

The Standard Model of particle theory containing specific gauge interactions is expected to have more structures when extended to energies above 1-10 TeV, where supersymmetry might be incorporated. Indeed, to derive the Standard Model as an effective theory from a unified theory containing gravity is one of the chief prospects of theoretical particle physics. One of the most important aspects of a supersymmetric gauge theory is that its vacuum, due to the omnipresence of scalars in the theory, can be highly nontrivial, as parametrized by the vacuum expectation values (VEVs) of gauge invariant operators (GIOs) composed of these scalar fields [1-3]. This vacuum moduli space (VMS) can be explicitly obtained as solution of constraints coming from F-flatness and D-flatness and be realized, in the language of algebraic geometry, as an algebraic variety [4].

Supersymmetric extensions of the Standard Model clearly constitute one of the central subjects in particle phenomenology. In particular, the minimal extension, the minimal supersymmetric standard model (MSSM), and its variants, have been subject to intense investigations. The flat directions of the MSSM have been identified in [5].

\footnotetext{
*Yan.Xiao@city.ac.uk

hey@maths.ox.ac.uk

*cyril.matti@a3.epfl.ch
}

Published by the American Physical Society under the terms of the Creative Commons Attribution 4.0 International license. Further distribution of this work must maintain attribution to the author(s) and the published article's title, journal citation, and DOI. Funded by SCOAP.
Combining these directions of thought, a long program was launched to study the vacuum geometry of the MSSM and its relatives [6-11]: under the guiding principle that "interesting geometry is coextensive with interesting physics," the ultimate goal is to use geometric and topological properties of VMS as a selection rule for operators in the Standard Model Lagrangian. Specifically, if the VMS were to be found to have some special form in the mathematical sense, which (1) cannot be explained in terms of symmetries relating the relevant degree of freedom in the low energy effective field theory; and (2) is very unlikely to have occurred by chance, then this special form should be regarded as a consequence of some unknown physics. In this setting, we take special to mean nontrivial properties of algebraic geometry, such as exhibited by interesting topological invariants or emergence of special holonomy.

Under such a spirit, the presence of any special geometry would be a collective consequence of factors such as gauge group, particle spectrum and the interactions within the theory. Therefore, if a special geometry is found within the low energy sector of a theory and this geometry is very unlikely to have arisen by chance, then the existence of such geometry in the VMS should be a fundamental property across all energy scales. Hence, the addition of higher dimensional operators to our theory can only occur when they are compatible with this structure. In such sense, we are placing very restrictive constraints on allowed physical processes that are mediated by certain operators.

Already, many interesting features have been found, such as the VMS of the electroweak sector being an affine cone over the classical Veronese surface, a structure ruined by addition of R-parity-violating operators, or the sensitive 
dependence of the geometry on the number of generations, or the appearance of Calabi-Yau varieties, etc., [9-11]. Supersymmetry and the VMS thus provide us with a low energy window of how geometry can guide certain phenomenological questions. A good analogy would be the study of complex numbers: many unforeseen and crucial properties of analytic functions are visible only after the complex extension of the real numbers, so too would important properties of quantum field theories-and the Standard Model in particular-only be visible after the supersymmetric extension. In this light, regardless of whether there exist supersymmetric particles, the study of supersymmetric structures and the VMS is an integral part of the study of field theory.

Despite the progress made over the years in the aforementioned program, an important question has remained: "what is the VMS of the MSSM?" The reason is purely computational: in component form, there are about 50 scalars and about 1000 GIOs, which by the state-of-the-art standard procedure in algebraic geometry adapted to calculate the VMS [10], is beyond the computational power of even the most sophisticated computers by direct means. Therefore, investigations thus far have focused on the electroweak sector wherein, as discussed, so much have already been uncovered. It is indeed expected that the geometry of the full MSSM would have far richer and salient features.

Rather fortuitously, there has been a parallel program in studying supersymmetric gauge theories: this is the socalled plethystic program $[12,13]$. It originated in the study of quiver gauge theories which arise from string theory, as world-volume theories on D-branes probing Calabi-Yau singularities [14], which have become the playground for the AdS/CFT correspondence [15]. Here, the VMS of the gauge theory is, by construction, the affine Calabi-Yau variety transverse to the $\mathrm{D}$-branes, and was in part the initial motivation for the VMS/phenomenology program. It allows one to build criteria to rule out certain top-down string model building to obtain desired low-energy outcomes: if the VMS is not Calabi-Yau, then one cannot use a direct top-down method.

The central object to the plethystic program is the Hilbert series, well-known to algebraic geometry as the generating function for counting the dimension of graded pieces of the coordinate ring. Harnessing this analogy with the superconformal index [16-23], the original motivation was to study the chiral ring of Bogomol'nyi-Prasad-Sommerfield (BPS) operators in supersymmetric gauge theories: the Hilbert series the counts the single-trace operators, whilst its plethystic exponential counts the multi-trace, and its plethystic logarithm encodes the generators and relations of the variety. A host of activity ensued, developing and refining various aspects of the program [24-36], even using the Hilbert-series technology to (the regular, nonsupersymmetric) Standard-Model phenomenology [37-40].

A natural question therefore arises as to whether the two programmes can come to a useful syzygy. Specifically, can certain properties of VMS for the MSSM be obtained without recourse to the computationally expensive elimination algorithm, but be deduced from the Hilbert series, which may be calculated via other means? Luckily, this is indeed the case. When the algebraic variety has extra symmetries, such as precisely in our cases, when they come from certain symplectic quotients of Lie-group invariants, there is a classic method of Molien-Weyl integration [41] to obtain the Hilbert series. The purpose of this paper is to perform this, albeit difficult, integral and obtain the Hilbert series explicitly for the MSSM, whence one can further deduce relevant geometrical quantities.

The objects that immediately follow from the Hilbert series are the degree and dimension of the VMS. In this work, we obtain the dimension of the VMS to be 40 and the degree given by the product of the following prime factors

$$
\begin{aligned}
& 2^{898} \cdot 3^{324} \cdot 5^{145} \cdot 7^{120} \cdot 11^{58} \cdot 13^{53} \cdot 17^{31} \cdot 19^{35} \cdot 23^{21} \cdot 29^{16} \cdot 31^{14} \cdot 37^{17} \cdot 41^{10} \cdot 43^{11} \cdot 47^{13} \cdot 53^{7} \cdot 59^{8} \\
& 61^{10} \cdot 67^{6} \cdot 71^{4} \cdot 73^{11} \cdot 79^{6} \cdot 83^{6} \cdot 89^{5} \cdot 97^{5} \cdot 101^{2} \cdot 103^{4} \cdot 107^{3} \cdot 109^{3} \cdot 113^{2} \cdot 127^{3} \cdot 131^{3} \cdot 137^{2} \cdot 139 . \\
& 149^{3} \cdot 151^{3} \cdot 157^{2} \cdot 163^{5} \cdot 167 \cdot 173 \cdot 179^{3} \cdot 181^{3} \cdot 191 \cdot 193^{5} \cdot 197^{2} \cdot 199 \cdot 211^{2} \cdot 229 \cdot 251 \cdot 257^{2} \cdot 263 \\
& 269 \cdot 271^{3} \cdot 277 \cdot 283^{2} \cdot 311 \cdot 313 \cdot 331 \cdot 337 \cdot 353 \cdot 373^{3} \cdot 379 \cdot 389 \cdot 431 \cdot 433 \cdot 443 \cdot 461 \cdot 467 \cdot 491 \\
& 509 \cdot 521 \cdot 541^{2} \cdot 547^{2} \cdot 557^{3} \cdot 563^{2} \cdot 587 \cdot 599 \cdot 607 \cdot 643^{3} \cdot 727 \cdot 757 \cdot 773^{3} \cdot 811 \cdot 821 \cdot 977 \cdot 1061 .
\end{aligned}
$$$$
1151 \cdot 1279 \cdot 1531 \cdot 1549 \cdot 1571 \cdot 1579 \text {, }
$$

with the following number that does not contain any prime factors between 1579 and number of order $10^{11}{ }^{1}$

\footnotetext{
${ }^{1}$ This number itself is not necessarily a prime but factorization of this number beyond primes of order $10^{11}$ is out of the capabilities of normal laptop.
} 
11196186329560947241455148908824054684468743740728908934170824241971190830192362923

499173415152896923453222633515598618927623333905606780549950560718456751981495721350

72426809129738886481086808646498896030206338463083506395355860193483377616149055621

18460688361209927517001930882524733517050667597849316746015467332076233464803805765

04692754875602278133281298256355246484194394745536495217014696615305675124055952742

70528682628820988014324086631325598762474204655322926194264597267995454863446388500

79630506164699.

The organization of the paper is as follows. Section II reviews some elements of the plethystic program and we can see therein how it establishes the connection between Hilbert series and the geometry of the VMS. Section III gives examples illustrating the program both in SQCD and Abelian gauge theory. Section IV establishes the scene for the plethystic integral for the MSSM using characters of $S U(3), S U(2)$ and $U(1)$ as well as corresponding charges for the matter content thereof. Section V gives the description of obtaining the Hilbert series for the MSSM with certain subtleties and the main obstacles within this procedure. The results are also presented with more details in this section as well.

Lastly, the VMS obtained here is not constrained by the superpotential $W$ of the MSSM, i.e., the relations from requiring $\partial W / \partial \phi_{i}=0$ with $\phi_{i}$ being the scalar component of the chiral fields in the MSSM, are not imposed on reaching the VMS. The case of nontrivial superpotential $W \neq 0$ is therefore left for future work.

\section{THE PLETHYSTIC PROGRAM}

In this section, we review some aspects of the plethystic program. The reader is also referred to [42] for a rapid review of the program and its context within quiver representations and gauge theory.

\section{A. Elimination algorithm for VMS}

We first briefly recall the algorithm for computing the VMS of a generic $\mathcal{N}=1$ supersymmetric gauge theory with gauge group $\mathcal{G}$, fields whose scalar components are $\phi_{i}$, and a polynomial superpotential $W$ therein. The most efficient method to obtain the VMS is as follows.

(1) INPUT:

(a) Superpotential $W\left(\left\{\phi_{i}\right\}\right)$, a polynomial in variables $\phi_{i=1, \ldots, n}$.

(b) Generators of GIOs: $r_{j}\left(\left\{\phi_{i}\right\}\right), j=1, \ldots, k$ polynomials in $\phi_{i}$ invariant under $\mathcal{G}$.

(2) ALGORITHM:

(a) Define the polynomial ring $R=\mathbb{C}\left[\phi_{i=1, \ldots, n}\right.$, $\left.y_{j=1, \ldots, k}\right]$.

(b) Consider the ideal $I=\left\langle\frac{\partial W}{\partial \phi_{i}}, y_{j}-r_{j}\left(\left\{\phi_{i}\right\}\right)\right\rangle$. (c) Eliminate all variables $\phi_{i}$ from $I \subset R$, giving the ideal $\mathcal{M}$ in terms of $y_{j}$.

(3) OUTPUT:

$\mathcal{M}$ corresponds to the VMS as an affine variety in $\mathbb{C}\left[y_{1}, \ldots, y_{k}\right]$.

In the ensuing, we will address general varieties $\mathcal{X}$ though ultimately we will specialize to when $\mathcal{X}$ is obtained as the VMS $\mathcal{M}$ from the above.

\section{B. The Hilbert series}

DEFINITION 2.1: Given an algebraic variety $\mathcal{X}$ in $\mathbb{C}\left[x_{1}, \ldots, x_{n}\right]$, the Hilbert series is the generating function of the graded pieces

$$
H_{\mathcal{X}}(t)=\sum_{i=0}^{\infty}\left(\operatorname{dim}_{\mathbb{C}} \mathcal{X}_{i}\right) t^{i}
$$

where $\mathcal{X}_{i}$ the $i$ th graded piece of the coordinate ring for $\mathcal{X}$ and can be regarded as the number of independent degree $i$ (Laurent) polynomials on $\mathcal{X}$.

Note that the Hilbert series is not a topological invariant and it depends on the embedding of $\mathcal{X}$. Of course, $H(t)$ can be generalized to be multivariate $H\left(t_{1}, \ldots, t_{n}\right)$ by considering the multigraded pieces $\mathcal{X}_{i_{1}, \ldots, i_{n}}$. The dummy variables $t_{i}$ are called fugacities in the physics literature. When there is more than one variable $t_{i}$, the Hilbert series is called refined, otherwise it is often called unrefined.

There are two important forms of Hilbert series which will be used later in this paper for obtaining the degree and dimension of the underlying variety. We have that (cf. [43,44])

Theorem 2.1: The Hilbert series $H(t)$ is a rational function in $t$ and can be written in two ways:

$H_{\mathcal{X}}(t)= \begin{cases}\frac{Q(t)}{(1-t)^{k}}, & \text { Hilbert series of the first kind; } \\ \frac{P(t)}{(1-t)^{\operatorname{dim}(\mathcal{X})}}, & \text { Hilbert series of the second kind. }\end{cases}$

Here both $P(t)$ and $Q(t)$ are polynomials with integer coefficients and the dimension of the embedding space is 
given by the power of the denominators. Moreover, $P(1)=\operatorname{degree}(\mathcal{X})$.

Thus we have a convenient way to obtain the degree of the variety. ${ }^{2}$

Furthermore, since the Hilbert series is a rational function, we have

Theorem 2.2: $H(t)$ affords a partial-fraction expansion around $t=1[45,46]$

$$
H_{\mathcal{X}}(t)=\frac{P(1)}{(1-t)^{\operatorname{dim}(\mathcal{X})}}+\cdots .
$$

Thus, the coefficient of the leading pole gives the degree of the variety while the order of the pole is the dimension.

Indeed, for Calabi-Yau varieties, the coefficient of the leading pole can also be interpreted as the volume of a base Sasaki-Einstein manifold, which in the AdS/CFT context is related to the central charges of the supersymmetric gauge theory [30,46-48].

Remark: We remark that when $\mathcal{X}$ is a quotient variety, i.e., $\mathcal{X} \simeq \mathbb{C}^{n} / \Gamma$ for some discrete finite group $\Gamma$ acting on the $n$ coordinates of $\mathbb{C}^{n}$, the problem of computing $H\left(t ; \mathbb{C}^{n} / \Gamma\right)$ reduces to counting the number of algebraically independent polynomials of each degree that are invariant under group action. This problem was solved by Molien $[43,49]$ and the corresponding Hilbert series is the wellknown Molien series, which can be computed by a sum over group elements:

$$
H_{\mathcal{X}}(t)=\frac{1}{|\Gamma|} \sum_{g \in \Gamma} \operatorname{det}(\mathbb{I}-t g)^{-1}
$$

\section{Molien-Weyl formula}

The case of our principle interest is when $\mathcal{X}$ is not a finite quotient, but of the form of a symplectic quotient by a (continuous) Lie group coming from the gauge symmetry. Luckily, there is a generalization of (2.4) into a so-called Molien-Weyl integral [41] (cf. [8,24,25]). The problem of finding invariants under continuous gauge group is at the heart of invariant theory that can be traced back to the 19th century and we present a rapid review of the origin of Hilbert series and Gröbner bases in the context of commutative algebra in Appendix C.

For our incarnation in physics, we wish to compute the Hilbert series for $\mathcal{X}=\mathcal{M}$ coming from the algorithm in Sec. II A, whose coordinate ring $\mathcal{R}$ is the projection of the quotient ring $R / I$ onto $\mathbb{C}\left[y_{j}\right]$. Now, the complexified gauge group $\mathcal{G}_{c}$ and global symmetry group act naturally on $\mathcal{R}$

\footnotetext{
${ }^{2}$ Recall that when an ideal is a single polynomial, i.e., $\mathcal{X}$ is a hypersurface, the degree of the variety is simply the degree of the polynomial. For multiple polynomials, the degree is a generalization of this notion. It then becomes the number of intersection points between a generic line and the variety.
}

and we can grade the elements therein with gauge and global charges. Let us denote the global Abelian charge as $t_{i}$ and the Cartan subgroup of the gauge group $\mathcal{G}_{v}$ by $z_{i}$. This gives the generating function, i.e., the Hilbert series, of the graded ring $\mathcal{R}$ as

$$
H_{\mathcal{R}}(t ; z)=\sum_{n, m} a_{n m} z^{n} t^{m}
$$

which can be written as a power series in the global charge $t$ and a Laurent expansion in the gauge charges $z$. Since the gauge symmetry commutes with global symmetries, all elements of $\mathcal{R}$ with given charge $t^{m}$ should form a representation $\chi^{m}$ of $\mathcal{G}_{c}$. Therefore, the coefficients of $t^{n}$ in Eq. (2.5) is the character of a $\mathcal{G}_{c}$ representation,

$H_{\mathcal{R}}(t, z)=\sum_{m=0}^{\infty} \chi^{m}(z) t^{m}=\sum_{m=0}^{\infty}\left(\sum_{i} a_{i}^{m} \chi^{(i)}(z)\right) t^{m}$.

In the last step, we have decomposed $\chi^{m}$, the representation on the elements of charge $t^{m}$, into irreducible representations $\chi^{(i)}$. Therefore, the generating function for invariants is given by the projection onto the trivial representation with character $\chi^{(0)}=1$,

$$
H_{\mathcal{R}}^{\mathrm{inv}}(t)=\sum_{m=0}^{\infty} a_{0}^{m} t^{m}
$$

The projection is done by averaging $H(t ; z)$ on the gauge group with Haar measure $d \mu(z)$,

$$
\int d \mu(z) \chi^{(i)}(z)=\delta_{i, 0}
$$

Explicitly, a group $G$ of rank $r$ has its Haar measure in terms of contour integral

$$
\frac{1}{|W|} \prod_{i=1}^{r} \oint_{\left|z_{i}\right|=1} \frac{d z_{i}}{2 \pi i z_{i}} \prod_{\alpha \in \Delta}\left(1-\prod_{i}^{r} z_{i}^{\alpha_{i}}\right),
$$

where $|W|$ is the order of the Weyl group $W$ of $\mathrm{G}$ and $\alpha$ is a root, or the weight of the adjoint representation such that $\alpha_{i}$ is the $i$ th entry of the weight vector in the Dynkin basis.

Putting all the above together, the Molien-Weyl formula for the Hilbert series of the variety $\mathcal{M}$ whose coordinate ring is $\mathcal{R}$ reads

$H_{\mathcal{R}}^{\mathrm{inv}}(t)=\frac{1}{|W|} \prod_{i=1}^{r} \oint_{\left|z_{i}\right|=1} \frac{d z_{i}}{2 \pi i z_{i}} \prod_{\alpha \in \Delta}\left(1-\prod_{i}^{r} z_{i}^{\alpha_{i}}\right) H_{\mathcal{R}}(t ; z)$.

Note that the integration requires knowledge about the Hilbert series of the coordinate ring $\mathcal{R}$. 


\section{Plethystics and syzygies}

The next crucial concept needed is that of plethystics.

DEFINITION 2.2: Let $g\left(t_{1}, \ldots, t_{n}\right)$ be a multivariate analytic function. The plethystic exponential (PE) is

$\operatorname{PE}\left[g\left(t_{1}, \ldots, t_{n}\right)\right]:=\exp \left(\sum_{k=1}^{\infty} \frac{g\left(t_{1}^{k}, \ldots, t_{n}^{k}\right)-g(0, \ldots, 0)}{k}\right)$.

It is easy to show (q.v., [13]) that (being an exponential) the plethystic exponential is multiplicative in additive arguments, and furthermore

$$
\begin{aligned}
f(t) & =\sum_{n=0}^{\infty} a_{n} t^{n} \\
\Rightarrow P E[f(t)] & =\exp \left(\sum_{n=1}^{\infty} \frac{f\left(t^{n}\right)-f(0)}{n}\right) \\
& =\prod_{n=1}^{\infty}\left(1-t^{n}\right)^{-a_{n} .}
\end{aligned}
$$

The product form is particularly useful and it is usually called Euler form.

It is a nontrivial fact $[13,41]$ that this has an analytic inverse function called the plethystic logarithm

$\mathrm{PE}^{-1}\left[g\left(t_{1}, \ldots, t_{n}\right)\right]=\sum_{k=1}^{\infty} \frac{\mu(k)}{k} \log \left(g\left(t_{1}^{k}, \ldots, t_{n}^{k}\right)\right)$,

where

$\mu(k):= \begin{cases}0 & k \text { has repeated prime factors } \\ 1 & k=1 \\ (-1)^{n} & k \text { is a product of } n \text { distinct primes }\end{cases}$

is the Möbius mu-function.

Remarkably, the plethystic logarithm can be used to find the defining relation (syzygies) of the generators of an algebraic variety $[12,13]$.

PROPOSITION 2.1: Given Hilbert series $H(t ; \mathcal{X})$ of an algebraic variety $\mathcal{X}$, the plethystic logarithm is of the form

$$
\mathrm{PE}^{-1}[H(t ; \mathcal{M})]=b_{1} t+b_{2} t^{2}+b_{3} t^{3}+\cdots
$$

where all $b_{n} \in \mathbb{Z}$ and a positive $b_{n}$ corresponds to a generator in coordinate ring of $\mathcal{X}$ and a negative $b_{n}$, a relation. In particular, if $\mathcal{X}$ is complete intersection, then $P E^{-1}[H(t ; \mathcal{M})]$ is a finite polynomial.

We illustrative this proposition in detail with concrete examples in Appendix A.
Lastly, we would like to see the connection between PE and Hilbert series $H_{\mathcal{R}}$ through a quick demonstration. We first show how $H_{\mathcal{R}}$ defined in Sec. II C is calculated and thus linked to PE with a toy example. Now we consider a theory of $n$ free chiral fields $\phi_{1}, \ldots, \phi_{n}$ with $U(n)$ global symmetry. Therefore, we have a collection of maximally commuting $U(1)$ 's in the $U(n)$ and each $U(1)$ has its corresponding charge fugacity $t_{i}$ to the field $\phi_{i}$ (with $i=1, \ldots, n)$. Chiral operators are thus described by $\phi_{1}^{k_{1}} \cdots \phi_{n}^{k_{n}}$ (with $k_{1}, \ldots, k_{n}=0,1,2, \ldots$ ). The Hilbert series $H_{\mathcal{R}}$ for such theory is therefore

$$
H_{\mathcal{R}}=\sum_{k_{1}, \ldots, k_{n}=0}^{\infty} t_{1}^{k_{1}} \cdots t_{n}^{k_{n}}=\prod_{i=1}^{n} \frac{1}{1-t_{i}} .
$$

Now we can rewrite the fugacities $t_{i}$ as

$$
t_{1}=t x_{1}, \quad t_{2}=t \frac{x_{2}}{x_{1}}, \quad t_{3}=t \frac{x_{3}}{x_{2}}, \ldots, t_{n-1}=t \frac{x_{n-1}}{x_{n-2}}, \quad t_{n}=\frac{t}{x_{n-1}},
$$

where $x_{1}, \ldots, x_{n-1}$ are $S U(n)$ fugacities and $t$ is the $U(1)$ fugacity. With such substitution, we have

$$
\begin{aligned}
& H_{\mathcal{R}}\left(t ; x_{1}, x_{2}, \ldots, x_{n-1}\right) \\
& \quad=1+\underbrace{\left(x_{1}+\frac{x_{2}}{x_{1}}+\cdots+\frac{x_{n-1}}{x_{n-2}}+\frac{1}{x_{n-1}}\right)}_{\text {the character of the fundamental rep of } S U(n)}+\cdots
\end{aligned}
$$

If we denote the Dynkin label of the character of an irrep of $S U(n)$ by $\left[k_{1}, \ldots, k_{n-1}\right]$, we have

$$
\begin{aligned}
H_{\mathcal{R}}\left(t ; x_{1}, x_{2}, \ldots, x_{n-1}\right) & =\sum_{k=0}^{\infty}[k, 0, \ldots, 0] t^{k} \\
& \equiv \operatorname{PE}[[1,0, \ldots, 0] t],
\end{aligned}
$$

where $[k, 0, \ldots, 0]$ is the $k$ th symmetric product of the fundamental representation. From the definition of PE in Eq. (2.11), we see that indeed both the PE and $H_{\mathcal{R}}$ in our example generate the symmetrization of their arguments. In particular, if we input $[1,0, \ldots, 0] t$ into the PE, we obtain the symmetric products in each term of the series: $\operatorname{PE}[[1,0, \ldots, 0] t]=\sum_{k=0}^{\infty}[k, 0, \ldots, 0] t^{k}$.

\section{E. Summary}

To summarize, the general expression of the Hilbert series $[8,24]$ in terms of the PE of the character is

$$
\begin{aligned}
\operatorname{PE}\left[\chi_{R}^{G}\left(z_{a}\right) \sum_{i}^{N_{f}} t_{i}\right] & =H_{\mathcal{R}}\left(t_{i}, z_{a}\right) \\
& \equiv \exp \left[\sum_{k=1}^{\infty} \sum_{i=1}^{N_{f}} \frac{1}{k}\left(t_{i}^{k} \chi_{R}^{G}\left(z_{a}^{k}\right)\right)\right],
\end{aligned}
$$


where $\chi_{R}^{G}\left(t_{i}, z_{a}\right)$ is the character for representation $R$ of group $G$ and it is expanded into monomials of complex variables $z_{i}$. Note that the number of complex variables $z_{a}$ is equal to the rank of group $G{ }^{3}$ The expansion of the PE gives the complete set of combinations of "fugacities" $t_{i}$. To find the generating function of GIOs under group $G$, we need to project the representation generated by the PE onto trivial subrepresentations of $G$. This is can be carried out by integrating over the whole group. This is precisely the Hilbert series $H_{\mathcal{R}}^{\text {inv }}$ in Eq. (2.10) whose Molien-Weyl formula for plethystic integral is given by

$g=\int_{G} \mathrm{~d} \mu_{G} \mathrm{PE}\left[\chi_{R}^{G}\left(z_{a}\right) \sum_{i}^{N_{f}} t_{i}\right]=\int_{G} \mathrm{~d} \mu_{G} H_{\mathcal{R}}\left(t_{i}, z_{a}\right)$,

where $\mathrm{d} \mu_{G}$ is the Haar measure for group $G$. With these data at hand, we conveniently package them into the following theorem:

Theorem 2.3 (Molien-Weyl integral for the Hilbert series): Given gauge group $G$, with Haar measure $\int_{G} \mathrm{~d} \mu_{G}$ and corresponding plethystic exponential defined in Eq. (2.15), the Hilbert series is computed by the following formula

$$
\begin{aligned}
g=H_{\mathcal{R}}^{\mathrm{inv}}\left(t_{i}\right) & =\int_{G} \mathrm{~d} \mu_{G} H_{\mathcal{R}}\left(t_{i}, z_{a}\right) \\
& =\int_{G} \mathrm{~d} \mu_{G} \operatorname{PE}\left[\chi_{R}^{G}\left(z_{a}\right) \sum_{i}^{N_{f}} t_{i}\right],
\end{aligned}
$$

where $\chi_{R}^{G}\left(t_{i}, z_{a}\right)$ is the character for representation $R$ of group $G$ and it is expanded into monomials of complex variables $z_{i}$ and the Haar measure is given by

$$
\int_{G} \mathrm{~d} \mu_{G}=\frac{1}{|W|} \prod_{i=1}^{\operatorname{rank}(G)} \int_{\left|z_{i}\right|=1} \frac{d z_{i}}{2 \pi i z_{i}} \prod_{\alpha \in \Delta}\left(1-\prod_{i}^{\operatorname{rank}(G)} z_{i}^{\alpha_{i}}\right),
$$

where $W$ is the Weyl group and $\alpha$ is a root, or the weight of the adjoint representation such that $\alpha_{i}$ is the $i$ th entry of the weight vector in the Dynkin basis.

The remainder of this paper will be used to evaluate this integral explicitly, first for some warm-up cases, and ultimately for the MSSM itself.

\section{WARM-UP EXAMPLES}

Our goal is to apply the technology introduced in Sec. II to the MSSM, with gauge group $\mathcal{G}=S U(3) \times S U(2) \times U(1)$. Before doing so, let us warm up with two illustrative examples: (1) the SQCD sector and (2) a single Abelian

\footnotetext{
${ }^{3}$ The variable $N_{f}$ in the expression is the total number of flavors in terms of gauge theory language.
}

gauge theory. This will give us a more concrete understanding of all the previous definitions from physical side.

\section{A. SQCD}

Let us look at the example of SQCD with $N_{c}$ colors and $N_{f}$ flavors, but without superpotential [8]. Here, the GIOs are symmetric combinations of quarks and antiquarks, transforming in the bifundamental $[1,0, \ldots, 0 ; 0, \ldots, 0,1]$ of $S U\left(N_{f}\right)_{L} \times S U\left(N_{c}\right)$ and the bifundamental $[1,0, \ldots, 0$; $0, \ldots, 0,1]$ of $S U\left(N_{c}\right) \times S U\left(N_{f}\right)_{R}$ respectively. This is the quark sector of the calculation which we are about to perform.

In the above, we have used the standard Young diagram for irreducible representations of $S U(n)$. Let $\lambda_{i}$ be the length of the $i$ th row $(1 \leq i \leq n-1)$ and let $a_{i}=\lambda_{i}-\lambda_{i+1}$ be the differences of lengths of rows. In such notation, we have a representation written as $\left[a_{1}, a_{2}, \ldots, a_{n-1}\right]$, of length $n-1$. For example, $[1,0, \ldots, 0]$ represents the fundamental representation, $[0, \ldots, 0,1]$ represents the antifundamental representation, and $[1,0, \ldots, 0,1]$ (the second 1 is at the $(n-1)$ th position represents the adjoint representation). For a product gauge group $S U(n) \times S U(n)$, we use notation $[\ldots ; \ldots]$ where the $(n-1)$-tuple to the left of the semicolon is the representation for the left $S U(n)$, and vice versa on the right. Finally, let us denote the character for the (anti) fundamental representation of $S U(N)$ as $\chi_{[0, \ldots, 1]}^{S U(N)}$ and $\chi_{[1,0, \ldots, 0]}^{S U(N)}$ respectively.

To use the Weyl-Molien formula, we need to introduce weights for elements in the Cartan subgroup for different groups. We use $z_{a}, a=1, \ldots, N_{c}-1$ for color weights and $t_{i}, \tilde{t}_{i}, i=1, \ldots, N_{f}$ for flavor weights. Therefore, the character for a quark becomes $\chi_{[1,0, \ldots, 0 ; 0, \ldots, 0,1]}^{S U\left(N_{f}\right)_{L} \times S U\left(N_{c}\right)}\left(t_{i}, z_{a}\right)$ and that for an antiquark is $\chi_{[1,0, \ldots, 0 ; 0, \ldots, 0,1]}^{S U\left(N_{c}\right) \times S U\left(N_{f}\right)_{R}}\left(\tilde{t}_{i}, z_{a}\right)$. We further introduce two more variables for counting number of quarks and antiquarks $t$ and $\tilde{t}$ respectively. The plethystic exponential from (2.11) is precisely the object which constructs symmetric products of quarks and antiquarks:

$$
\begin{aligned}
\operatorname{PE}\left[t \chi_{[1,0, \ldots, 0 ; 0, \ldots, 0,1]}\right. & S U\left(N_{f}\right)_{L} \times S U\left(N_{c}\right) \\
\equiv & \exp \left[\sum _ { k = 0 } ^ { \infty } \frac { 1 } { k } \left(t^{k} \chi_{[1,0, \ldots, 0 ; 0, \ldots, 0,1]}^{S U\left(N_{f}\right)_{L} \times S U\left(N_{c}\right)}\left(t_{i}^{k}, z_{a}^{k}\right)\right.\right. \\
& +\sum_{k=0}^{\infty} \frac{1}{k}\left(\tilde{t}^{k} \chi_{[1,0, \ldots, \ldots, 0 ; 0, \ldots, 0,1]}^{S U\left(N_{c}\right) \times S U\left(N_{f}\right)_{R}}\left(\tilde{t}_{i}, z_{a}\right)\right] \\
&
\end{aligned}
$$

Expanding the character more explicitly as

$$
{ }^{t} \chi_{[1,0, \ldots, 0 ; 0, \ldots, 0,1]}^{S U\left(N_{f}\right)_{L} \times S U\left(N_{c}\right)}\left(t_{i}, z_{a}\right)=\chi_{[0, \ldots, 0,1]}^{S U\left(N_{c}\right)}\left(z_{l}\right) \sum_{i=1}^{N_{f}} t_{i},
$$

gives us 


$$
\begin{aligned}
& \operatorname{PE}\left[\chi_{[1,0, \ldots, 0]}^{S U\left(N_{c}\right)}\left(z_{l}\right) \sum_{i=1}^{N_{f}} \tilde{t}_{i}+\chi_{[0, \ldots, 0,1]}^{S U\left(N_{c}\right)}\left(z_{l}\right) \sum_{j=1}^{N_{f}} t_{j}\right] \\
& =\exp \left[\sum_{k=0}^{\infty}\left(\frac{\chi_{[1,0, \ldots, 0]}^{S U\left(N_{c}\right)}\left(z_{l}^{k}\right) \sum_{i=1}^{N_{f}} \tilde{t}_{i}^{k}+\chi_{[0, \ldots, 0,1]}^{S U\left(N_{c}\right)}\left(z_{l}^{k}\right) \sum_{j=1}^{N_{f}} \tilde{t}_{j}^{k}}{k}\right)\right] .
\end{aligned}
$$

Here we associated dummy variables $t$ and $\tilde{t}$ to stand for quarks and antiquarks counting the global $U(1)$ charges in the maximal torus of the global symmetry. Therefore, we should restrict the values of $t_{i}$ to be $\left|t_{i}\right|<1$ for all $i$.

As described in Sec. II C, we want gauge invariant quantities, therefore, it is important that we project these representations onto trivial subrepresentations that are made up by quantities invariant under the action of a gauge group. The Molien-Weyl integral from (2.10) thus gives the requisite Hilbert series (generating function) for $\left(N_{f}, N_{c}\right)$ as

$$
\begin{aligned}
g^{\left(N_{f}, N_{c}\right)}= & \int_{S U\left(N_{c}\right)} \mathrm{d} \mu_{S U\left(N_{c}\right)} \\
& \times \operatorname{PE}\left[\chi_{[1,0, \ldots, 0]}^{S U\left(N_{c}\right)}\left(z_{l}\right) \sum_{i=1}^{N_{f}} \tilde{t}_{i}+\chi_{[0, \ldots, 0,1]}^{S U\left(N_{c}\right)}\left(z_{l}\right) \sum_{j=1}^{N_{f}} t_{j}\right] .
\end{aligned}
$$

The Haar measure $\mathrm{d} \mu_{S U\left(N_{c}\right)}$ can be explicitly written using Weyl's integration formula as (see, e.g., Sec. 26.2 of [41])

$$
\begin{aligned}
& \int_{S U\left(N_{c}\right)} \mathrm{d} \mu_{S U\left(N_{c}\right)} \\
& =\frac{1}{(2 \pi i)^{N_{c}-1} N_{c} !} \oint_{\left|z_{l}\right|=1} \prod_{l=1}^{N_{c}-1} \frac{\mathrm{d} z_{l}}{z_{l}} \Delta(\phi) \Delta\left(\phi^{-1}\right),
\end{aligned}
$$

where $\phi_{a}\left(z_{1}, \ldots, z_{N_{c}-1}\right)_{a=1}^{N_{c}}$ are the coordinates on the maximal torus of $S U\left(N_{c}\right)$ with $\prod_{a=1}^{N_{c}} \phi_{a}=1$, and $\Delta(\phi)=$ $\prod_{1 \leq a \leq b \leq N_{c}}\left(\phi_{a}-\phi_{b}\right)$ is the Vandermonde determinant.

Finally, let us construct the characters in the plethystic exponential. First we take the weights of the fundamental representation of $S U\left(N_{c}\right)$ to be

$$
\begin{aligned}
L_{1} & =(1,0, \ldots, 0), \quad L_{k}(0,0, \ldots,-1,1, \ldots, 0), \\
L_{N_{c}} & =(0, \ldots,-1),
\end{aligned}
$$

where all $L$ 's are $\left(N_{c}-1\right)$-tuples, and $L_{k}\left(2 \leq k \leq N_{c}-1\right)$ has -1 in the $(k-1)$ th position and 1 in the $k$ th position. With this particular choice of weights, the coordinates on the maximal torus of $S U\left(N_{c}\right)$ are given by

$$
\phi_{1}=z_{1}, \quad \phi_{k}=z_{k-1}^{-1} z_{k}, \quad \phi_{N_{c}}=z_{N_{c}-1}^{-1},
$$

with $2 \leq k \leq N_{c}-1$. Hence, the characters of the fundamental and antifundamental representations are

$$
\begin{aligned}
\chi_{[1,0, \ldots, 0]}^{S U\left(N_{c}\right)} & =\sum_{a=1}^{N_{c}} \phi_{a}=z_{1}+\sum_{k=1}^{N_{c}-1} \frac{z_{k}}{z_{k-1}}+\frac{1}{z_{N_{c}-1}}, \\
\chi_{[0,0, \ldots, 1]}^{S U\left(N_{c}\right)} & =\sum_{a=1}^{N_{c}} \phi_{a}^{-1}=\frac{1}{z_{1}}+\sum_{k=1}^{N_{c}-1} \frac{z_{k-1}}{z_{k}}+z_{N_{c}-1}
\end{aligned}
$$

Thus, we have that [8]:

Theorem 3.1: The final expression for the Hilbert series for SQCD is the ordinary integral

$$
\begin{aligned}
g^{\left(N_{f}, N_{c}\right)}\left(t_{i}, \tilde{t}_{i}\right)= & \frac{1}{(2 \pi i)^{N_{c}-1} N_{c} !} \oint_{\left|z_{l}\right|=1} \prod_{l=1}^{N_{c}-1} \frac{d z_{l}}{z_{l}} \Delta(\phi) \Delta\left(\phi^{-1}\right) \\
& \times \operatorname{PE}\left[\left(z_{1}+\sum_{k=2}^{N_{c}-1} \frac{z_{k}}{z_{k-1}}+\frac{1}{z_{N_{c}-1}}\right) \sum_{i=1}^{N_{f}} t_{i}\right. \\
& \left.+\left(\frac{1}{z_{1}}+\sum_{k=2}^{N_{c}-1} \frac{z_{k-1}}{z_{k}}+z_{N_{c}-1}\right) \sum_{j=1}^{N_{f}} \tilde{t}_{j}\right] .
\end{aligned}
$$

Note that this a refined Hilbert series in the $2 N_{f}$ variables $t_{i}$ an $\tilde{t}_{i}$.

\section{B. An Abelian gauge theory}

We have reviewed in the previous subsection, a rather formal and general example to elucidate the contents of Molien-Weyl formula for SQCD with $S U(N)$ gauge group. However, the spirit of the integral can be captured by a simple example using $U(1)$ without loss of generality [38]. First, consider a single complex scalar field charged under a $U(1)$ symmetry, i.e., $\phi \rightarrow e^{i \theta} \phi, \phi^{*} \rightarrow e^{-i \theta}$. Clearly, the gauge invariants are now $\left(\phi \phi^{*}\right)^{n}$ and there is only one such operator for each $n$. We can then define a formal series as

$$
H=\sum_{n=1}^{\infty} c_{n}\left(\phi \phi^{*}\right)^{n},
$$

where $c_{n}=1$ counts the number of different invariants of a given $n$ (since there is clearly only one per degree), so when expanded, it is

$$
H=1+\left(\phi \phi^{*}\right)+\left(\phi \phi^{*}\right)^{2}+\left(\phi \phi^{*}\right)^{3}+\cdots
$$

If $\left(\phi, \phi^{*}\right)$ are formally treated as numbers less than one, it is simply a geometric series in variables $\left(\phi, \phi^{*}\right)$,

$$
H\left(\phi, \phi^{*}\right)=\frac{1}{1-\phi \phi^{*}} .
$$

Here, we obtain a refined Hilbert series in two variables; because the field itself is a complex scalar, we can identify the field with its own corresponding fugacity. 
Introducing another variable $\theta$, the same variable that parametrizes the $U(1),(3.12)$ can be rewritten as the integral

$$
H=\frac{1}{2 \pi} \int_{0}^{2 \pi} \frac{\mathrm{d} \theta}{\left(1-\phi e^{i \theta}\right)\left(1-\phi^{*} e^{-i \theta}\right)} .
$$

This reparametrization can be seen by series expanding $\left(1-\phi e^{i \theta}\right)^{-1}\left(1-\theta^{*} e^{-i \theta}\right)^{-1}$, which is $\left(1+\phi e^{i \theta}+\right.$ $\left.\left(\phi e^{i \theta}\right)^{2}+\cdots\right)\left(1+\phi^{*} e^{-i \theta}+\left(\phi^{*} e^{-i \theta}\right)^{2}+\cdots\right)$. By multiplying out and collect terms according to powers of $e^{i \theta}$, we see that the terms that are free of $e^{i \theta}$ are exactly the formal series we started with, i.e., $1+\left(\phi \phi^{*}\right)+$ $\left(\phi \phi^{*}\right)^{2}+\left(\phi \phi^{*}\right)^{3}+\cdots$. The terms with any number of factors of $e^{i \theta}$ or $e^{-i \theta}$ vanish upon the $\theta$ integration.

Making substitution $z=e^{i \theta}$, the $\mathrm{d} \theta$ integral becomes a contour integral around $|z|=1$.

$$
H=\frac{1}{2 \pi i} \oint_{|z|=1} \frac{\mathrm{d} z}{z} \frac{1}{(1-\phi z)\left(1-\phi^{*} z^{-1}\right)} .
$$

We can further reframe the second part of the integrand

$$
\begin{aligned}
& \frac{1}{(1-\phi z)\left(1-\phi^{*} z^{-1}\right)} \\
& =\exp \left[-\log (1-\phi z)-\log \left(1-\phi^{*} z^{-1}\right)\right] \\
& =\exp \left[\sum_{n=1}^{\infty} \frac{(\phi z)^{n}}{n}+\sum_{n=1}^{\infty} \frac{\left(\phi^{*} z^{-1}\right)^{n}}{n}\right] \\
& =\operatorname{PE}\left[\phi z+\phi^{*} z^{-1}\right] .
\end{aligned}
$$

To understand the previous lines, let us expand the LHS of Eq. (3.15), with $\phi$ and $\phi^{*}$ being small complex numbers. To cubic order in both fields we have

$$
\begin{aligned}
\frac{1}{(1-\phi z)\left(1-\phi^{*} z^{-1}\right)}= & 1+\phi \phi^{*}+\left(\phi \phi^{*}\right)^{2}+\left(\phi \phi^{*}\right)^{3}+\cdots+z\left(\phi+\phi\left(\phi \phi^{*}\right)+\phi\left(\phi \phi^{*}\right)^{2}+\cdots\right) \\
& +z^{2}\left(\phi^{2}+\phi^{2}\left(\phi \phi^{*}\right)+\cdots\right)+z^{3} \phi^{3}+\phi^{* 3} z^{-3}+z^{-2}\left(\phi^{* 2}+\phi^{* 2}\left(\phi \phi^{*}\right)+\cdots\right) \\
& +z^{1}\left(\phi^{*}+\phi^{*}\left(\phi \phi^{*}\right)+\phi^{*}\left(\phi \phi^{*}\right)^{2}+\cdots\right) .
\end{aligned}
$$

From this expansion, the terms with no factors of $z$ are the ones invariant under $U(1)$, which are picked out by the contour integral. Equation (3.16) shows that we can obtain the series of charge +1 by multiplying with $z^{-2}$ and that of charge -2 by multiplying with $z$. These results follow from the fact that the expansion has already generated all possible combinations of $\phi$ and $\phi^{*}$. In doing so, we implicitly used the reasoning behind (2.10). There are two underlying concepts running in parallel.

First, Eq. (3.15) generates all possible arrangements of the scalar fields, as graded by charge. Indeed, we see the natural emergence of the plethystic exponential, as the generating function of all symmetric combination of its argument. Second, the integration over $d \theta=\frac{d z}{i z}$ is the integration over the group manifold $U(1)$. This makes sense as we want group invariant quantities, so we have to "average" over group elements. When integrated over $d \theta$, any terms with nontrivial powers of $z=e^{i \theta}$ become integrals $d \theta e^{i n \theta}$ for some integer $n$. This is identically 0 since integral is from 0 to $2 \pi$. Hence, terms with no powers of $z$ remain and are $U(1)$ invariant.

\section{MOLIEN-WEYL INTEGRAL FOR THE MSSM}

In this section, we set up the scene for performing the Molien-Weyl integral to obtain the Hilbert series of the MSSM. Then, we will use the result to interpret geometrical properties of the VMS for the MSSM. We emphasize that the analysis will be done with the superpotential $W=0$.
The group $\mathcal{G}$ under consideration for the MSSM is, of course, the product gauge group $S U(3) \times S U(2)_{L} \times U(1)_{Y}$. The corresponding character for the product group is then also a product for individual factor group, following from the very definition of a group character. Indeed, for a given group $G$, we can associate any representation $R$ with a character $\chi_{R}: G \rightarrow \mathbb{C}$, where the map is defined to be the trace of any group element $g$ in representation $R$. Under this definition, the character for the direct sum and the product of the representations is given by $\chi_{R_{i} \oplus R_{j}}=\chi_{R_{i}}+\chi_{R_{j}}$ and $\chi_{R_{i} \otimes R_{j}}=\chi_{R_{i}} \chi_{R_{j}}$. Thus equipped, we simply need to input the particle contents with the corresponding representation for the product gauge group of the MSSM, along with the appropriate Haar measure for each factor group, to construct the integral in Theorem 2.3.

The particle content for the MSSM are well known and recapitulated in Table I. The characters for each factor group are taken from [24] and the relevant ones are presented in Table II. In addition, we note that the Haar measure $\mu_{G}$ for gauge group $G$ is given by [50]

$\int_{G} \mathrm{~d} \mu_{G}=\frac{1}{(2 \pi i)^{r}} \oint_{\left|z_{1}\right|=1} \cdots \oint_{\left|z_{r}\right|=1} \frac{\mathrm{d} z_{1}}{z_{1}} \cdots \frac{\mathrm{d} z_{r}}{z_{r}} \prod_{\alpha^{+}}\left(1-\prod_{l=1}^{r} z_{l}^{\alpha_{l}^{+}}\right)$,

where $\alpha^{+}$are positive roots of the Lie algebra of the gauge group and $r=\operatorname{rank} G$. Specifically, this definition of the Haar measure is different from that of Eq. (3.5) such that 
TABLE I. Minimal Supersymmetric Standard Model particle contents are given in the table, where the Representation column entries give information how each particle transform under the product gauge group. For example, the first row means the quark $Q$ transforms in fundamental representation $\mathbf{3}$ of $S U(3), \mathbf{2}$ of $S U(2)$ and has charge $\frac{1}{6}$ under $U(1)$. In addition, $\overline{\mathbf{3}}$ means antifundamental representation.

\begin{tabular}{lcrl}
\hline \hline Field & Multiplicity & Representation & \multicolumn{1}{c}{ SM Particle } \\
\hline$Q$ & 3 & $(\mathbf{3}, \mathbf{2})_{\frac{1}{6}}$ & Left-handed quark doublet \\
$U^{C}$ & 3 & $(\overline{\mathbf{3}}, \mathbf{1})_{-\frac{2}{3}}$ & Right-handed up-type anti-quark \\
$D^{C}$ & 3 & $(\overline{\mathbf{3}}, \mathbf{1})_{\frac{1}{3}}$ & Right-handed down-type anti-quark \\
$L$ & 3 & $(\mathbf{1}, \mathbf{2})_{-\frac{1}{2}}$ & Left-handed lepton doublet \\
$E^{C}$ & 3 & $(\mathbf{1}, \mathbf{1})_{1}$ & Right-handed anti-lepton doublet \\
$H_{u}$ & 1 & $(\mathbf{1}, \mathbf{2})_{\frac{1}{2}}$ & Higgs \\
$H_{d}$ & 1 & $(\mathbf{1}, \mathbf{2})_{-\frac{1}{2}}$ & Higgs \\
\hline \hline
\end{tabular}

TABLE II. The characters used for constructing the plethystic exponential of the MSSM.

\begin{tabular}{lc}
\hline$S U(3)$ fundamental & $\chi_{\mathbf{3}}^{S U(3)}\left(z_{a}\right)=z_{1}+\frac{z_{2}}{z_{1}}+\frac{1}{z_{2}}$ \\
$S U(3)$ antifundamental & $\chi_{\overline{3}}^{S U(3)}\left(z_{a}\right)=\frac{1}{z_{1}}+\frac{z_{1}}{z_{2}}+z_{2}$ \\
$S U(2)$ (anti) fundamental & $\chi_{\overline{2}}^{S U(2)}(y)=\chi_{\mathbf{2}}^{S U(2)}(y)=y+\frac{1}{y}$ \\
$U(1)$ & $\chi(x)_{Q}^{U(1)}(x)=x^{Q}$ \\
\hline
\end{tabular}

only the positive roots are used here. A more detailed discussion can be found in [8,24]. For example, the measure for $S U(2)$ given by Eq. (3.5)

$\int_{S U(2)} \mathrm{d} \mu_{S U(2)}(z) \rightarrow \frac{1}{2} \frac{1}{2 \pi i} \oint_{|z|=1} \frac{\mathrm{d} z}{z}\left(1-z^{2}\right)\left(1-z^{-2}\right)$, has the term with negative exponent on $z$ as compared to that in Table III. Since only positive roots are used here and Weyl renormalization is not required, the practical calculation will be extensively reduced. These two seemingly different measures can be explicitly checked in calculation and we have done this for the $S U(2)$ case, whose results are indeed the same. Finally, the Haar measures for each group [24] are presented in Table III.

With the above data, we now proceed to explicitly construct the Molien-Weyl integral in its plethystic form, which we will call PI, with the full MSSM content. This simply involves putting each chiral field into its correct representation and input its quantum number for associated character. To do this, we first tabulate the characters of the particle content within the MSSM in Table IV. We can then use the formula of plethystic exponential in Eq. (2.11) to obtain the integrand. For example, the exponent of the plethystic exponential for left-handed quarks is

$$
\begin{aligned}
& \log \left[y ^ { 9 } z _ { 1 } ^ { 6 } z _ { 2 } ^ { 6 } \left\{\left(y-Q_{1} \sqrt[6]{x} z_{1}\right)\left(1-Q_{1} \sqrt[6]{x} y z_{1}\right)\left(y-Q_{2} \sqrt[6]{x} z_{1}\right)\left(1-Q_{2} \sqrt[6]{x} y z_{1}\right)\left(y-Q_{3} \sqrt[6]{x} z_{1}\right)\left(1-Q_{3} \sqrt[6]{x} y z_{1}\right)\right.\right. \\
& \quad \times\left(z_{2}-Q_{1} \sqrt[6]{x} y\right)\left(z_{2}-Q_{2} \sqrt[6]{x} y\right)\left(z_{2}-Q_{3} \sqrt[6]{x} y\right)\left(y z_{2}-Q_{1} \sqrt[6]{x}\right)\left(y z_{2}-Q_{2} \sqrt[6]{x}\right)\left(y z_{2}-Q_{3} \sqrt[6]{x}\right) \\
& \left.\left.\quad \times\left(y z_{1}-Q_{1} \sqrt[6]{x} z_{2}\right)\left(z_{1}-Q_{1} \sqrt[6]{x} y z_{2}\right)\left(y z_{1}-Q_{2} \sqrt[6]{x} z_{2}\right)\left(z_{1}-Q_{2} \sqrt[6]{x} y z_{2}\right)\left(y z_{1}-Q_{3} \sqrt[6]{x} z_{2}\right)\left(z_{1}-Q_{3} \sqrt[6]{x} y z_{2}\right)\right\}^{-1}\right]
\end{aligned}
$$

which upon taking exponential gives us the argument inside the logarithm. The plethystic exponentials for the rest of the particle content can be obtained in a similar fashion. Thus we have the following proposition.

TABLE III. The Haar measure of the MSSM gauge groups. Note here that the Haar measures are different from those of $[8,25]$. The ones presented here only use positive roots and do not need Weyl group renormalization.

\begin{tabular}{lc}
\hline \hline Group & Haar Measure \\
\hline$S U(3)$ & $\int_{S U(3)} \mathrm{d} \mu_{S U(3)}=\frac{1}{(2 \pi i)^{2}} \oint_{\left|z_{1}\right|=1} \frac{\mathrm{d} z_{1}}{z_{1}} \oint_{\left|z_{2}\right|=1} \frac{\mathrm{d} z_{2}}{z_{2}}\left(1-z_{1} z_{2}\right)\left(1-\frac{z_{1}^{2}}{z_{2}}\right)\left(1-\frac{z_{2}^{2}}{z_{1}}\right)$ \\
$S U(2)$ & $\int_{S U(2)} \mathrm{d} \mu_{S U(2)}=\frac{1}{2 \pi i} \oint_{|y|=1} \frac{\mathrm{d} y}{y}\left(1-y^{2}\right)$ \\
$U(1)$ & $\int_{U(1)} \mathrm{d} \mu_{U(1)}=\frac{1}{2 \pi i} \oint_{|x|=1} \frac{\mathrm{d} x}{x}$ \\
\hline \hline
\end{tabular}


TABLE IV. The characters of the MSSM particles under the Standard Model gauge group $S U(3) \times S U(2)_{L} \times U(1)_{Y}$.

\begin{tabular}{lcc}
\hline \hline Particle & Label & Character under $S U(3) \times S U(2)_{L} \times U(1)_{Y}$ \\
\hline Left-handed quarks & $Q_{i}$ & $x^{\frac{1}{6}}\left(y+\frac{1}{y}\right)\left(z_{1}+\frac{1}{z_{2}}+\frac{z_{2}}{z_{1}}\right)$ \\
Right-handed up-type anti-quarks & $u_{i}$ & $x^{-2 / 3}\left(\frac{z_{1}}{z_{2}}+z_{2}+\frac{1}{z_{1}}\right)$ \\
Right-handed down-type anti-quark & $d_{i}$ & $x^{1 / 3}\left(\frac{z_{1}}{z_{2}}+z_{2}+\frac{1}{z_{1}}\right)$ \\
Left-handed lepton & $L_{i}$ & $x^{-1 / 2}\left(y+\frac{1}{y}\right)$ \\
Right-handed anti-lepton & $e_{i}$ & $x$ \\
Up-type Higgs & $H_{u}$ & $x^{1 / 2}\left(y+\frac{1}{y}\right)$ \\
Down-type Higgs & $H_{d}$ & $x^{-1 / 2}\left(y+\frac{1}{y}\right)$ \\
\hline \hline
\end{tabular}

PROPOSITION 4.1: The Hilbert series for the VMS of the MSSM (with zero superpotential) is given by

$$
\mathrm{PI}=\frac{1}{(2 \pi i)^{4}} \oint_{|x|=1} \oint_{|y|=1} \oint_{\left|z_{1}\right|=1} \oint_{\left|z_{2}\right|=1} \operatorname{PE}\left(x, y, z_{1}, z_{2}, Q_{i}, L_{i}, u_{i}, d_{i}, e_{i}, H_{u}, H_{d}\right)
$$

where $i=1,2,3$ and $\operatorname{PE}\left(x, y, z_{1}, z_{2}, Q_{i}, L_{i}, u_{i}, d_{i}, e_{i}, H_{u}, H_{d}\right)$ is given by

$$
\begin{aligned}
& \mathrm{PE}\left(x, y, z_{1}, z_{2}, Q_{i}, L_{i}, u_{i}, d_{i}, e_{i}, H_{u}, H_{d}\right)=-x^{9} y^{13}\left(y^{2}-1\right) z_{1}^{10}\left(z_{1}^{2}-z_{2}\right) z_{2}^{10}\left(z_{1} z_{2}-1\right)\left(z_{1}-z_{2}^{2}\right) \\
& \times\left[\left(y-H_{u} \sqrt{x}\right)\left(H_{d} y-\sqrt{x}\right)\left(\sqrt{x} y-H_{d}\right)\left(H_{u} \sqrt{x} y-1\right)\left(x e_{1}-1\right)\left(x e_{2}-1\right)\right. \\
& \times\left(x e_{3}-1\right)\left(\sqrt{x} y-L_{1}\right)\left(y L_{1}-\sqrt{x}\right)\left(\sqrt{x} y-L_{2}\right)\left(y L_{2}-\sqrt{x}\right)\left(\sqrt{x} y-L_{3}\right)\left(y L_{3}-\sqrt{x}\right) \\
& \times\left(\sqrt[3]{x} d_{1}-z_{1}\right)\left(\sqrt[3]{x} d_{2}-z_{1}\right)\left(\sqrt[3]{x} d_{3}-z_{1}\right)\left(x^{2 / 3} z_{1}-u_{1}\right)\left(x^{2 / 3} z_{1}-u_{2}\right)\left(x^{2 / 3} z_{1}-u_{3}\right) \\
& \times\left(y-\sqrt[6]{x} Q_{1} z_{1}\right)\left(\sqrt[6]{x} y Q_{1} z_{1}-1\right)\left(y-\sqrt[6]{x} Q_{2} z_{1}\right)\left(\sqrt[6]{x} y Q_{2} z_{1}-1\right)\left(y-\sqrt[6]{x} Q_{3} z_{1}\right) \\
& \times\left(\sqrt[6]{x} y Q_{3} z_{1}-1\right)\left(\sqrt[6]{x} y Q_{1}-z_{2}\right)\left(\sqrt[6]{x} y Q_{2}-z_{2}\right)\left(\sqrt[6]{x} y Q_{3}-z_{2}\right)\left(\sqrt[3]{x} d_{1} z_{1}-z_{2}\right) \\
& \times\left(\sqrt[3]{x} d_{2} z_{1}-z_{2}\right)\left(\sqrt[3]{x} d_{3} z_{1}-z_{2}\right)\left(x^{2 / 3} z_{2}-u_{1} z_{1}\right)\left(x^{2 / 3} z_{2}-u_{2} z_{1}\right)\left(x^{2 / 3} z_{2}-u_{3} z_{1}\right) \\
& \times\left(y z_{2}-\sqrt[6]{x} Q_{1}\right)\left(y z_{2}-\sqrt[6]{x} Q_{2}\right)\left(y z_{2}-\sqrt[6]{x} Q_{3}\right)\left(\sqrt[3]{x} d_{1} z_{2}-1\right)\left(\sqrt[3]{x} d_{2} z_{2}-1\right) \\
& \times\left(\sqrt[3]{x} d_{3} z_{2}-1\right)\left(y z_{1}-\sqrt[6]{x} Q_{1} z_{2}\right)\left(\sqrt[6]{x} y Q_{1} z_{2}-z_{1}\right)\left(y z_{1}-\sqrt[6]{x} Q_{2} z_{2}\right)\left(\sqrt[6]{x} y Q_{2} z_{2}-z_{1}\right) \\
& \left.\times\left(y z_{1}-\sqrt[6]{x} Q_{3} z_{2}\right)\left(\sqrt[6]{x} y Q_{3} z_{2}-z_{1}\right)\left(x^{2 / 3}-u_{1} z_{2}\right)\left(x^{2 / 3}-u_{2} z_{2}\right)\left(x^{2 / 3}-u_{3} z_{2}\right)\right]^{-1},
\end{aligned}
$$

where the fugacities $t_{i} \in\left\{Q_{i}, L_{i}, u_{i}, d_{i}, e_{i}, H_{u}, H_{d}\right\}$ are taken to be $\left|t_{i}\right|<1$ due to the fact that they count the $U(1)$ charges inside the maximal torus of the global symmetries.

As can be seen, even though we have reduced the problem of computing the Hilbert series to an ordinary contour integral with the help of Molien-Weyl formula, the result is still a formidable integral, involving an integrand which is a rational function with 8 factors in the numerator and 49 factors in the denominator. The remainder of this paper is concerned with simplifying this integral explicitly and obtaining geometrical information therefrom. Moreover, we remark that there are fractional powers in the integration variables which might upset the reader: after all, the final answer is a Hilbert series, which must be a rational function. We will show in the ensuing section that all fractional powers actually cancel or disappear in the course of the integration, as is required.

\section{OBTAINING THE MSSM HILBERT SERIES}

In the previous section, we constructed the contour integral to obtain Hilbert series of the MSSM in (4.5), which gives the generating function that counts the GIOs. As we can see, there are 4 variables $\left(x, y, z_{1}, z_{2}\right)$ that need to be integrated over and the pole structure of the integrand is quite complicated. Therefore, it would be illuminating to record the detailed steps in performing the integration and we shall see that the subtleties of the pole positions become important during the integration procedure. The full codes and results can be accessed form [51]. 


\section{A. Finding poles and residues}

The integration procedure can be carried out as follows. The intermediate results are too complicated to be presented in the text, or even in an appendix, but is available at the following link to the repository.

\section{The y integral}

We simply integrate over the variable $y$ over contour $|y|=1$. More specifically, according to the residue theorem, we calculate the residue for poles for $y$ inside the contour prescribed. The poles for $y$ are functions of other complex variables with variable $x$ having fractional power due to $1 / 6 U(1)$ charge of left-handed quarks. These fractional powers will become a main reason for the complexity of later parts of the integral. With the only requirement of fugacities with modulus smaller than 1 , the positions of these poles are completely determined, i.e., whether inside or outside the unit circle prescribed for variable $y$. In fact, there are only 14 such poles for $y$ :

$$
\begin{aligned}
& \frac{H_{d}}{\sqrt{x}}, \quad \frac{L_{1}}{\sqrt{x}}, \quad \frac{L_{2}}{\sqrt{x}}, \quad \frac{L_{3}}{\sqrt{x}}, \quad H_{u} \sqrt{x}, \quad Q_{1} \sqrt[6]{x} z_{1}, \quad Q_{2} \sqrt[6]{x} z_{1} \\
& Q_{3} \sqrt[6]{x} z_{1}, \quad \frac{Q_{1} \sqrt[6]{x}}{z_{2}}, \quad \frac{Q_{2} \sqrt[6]{x}}{z_{2}}, \quad \frac{Q_{3} \sqrt[6]{x}}{z_{2}}, \quad \frac{Q_{1} \sqrt[6]{x} z_{2}}{z_{2}}, \quad \frac{Q_{2} \sqrt[6]{x} z_{2}}{z_{1}}, \quad \frac{Q_{3} \sqrt[6]{x} z_{2}}{z_{1}} .
\end{aligned}
$$

For each of these 14 poles, the residue can be readily obtained. Normally, we would sum these 14 separate residues, put them under the same denominator and cancel any common factors between the initial denominator and numerator. However, this direct approach is already beyond computer package such as Mathematica. To get a taste of the complexities of the rational functions under discussion, let us present 2 of the 14 residues, all of which are complicated rational functions of similar complexity (again, the reader is referred to the above url for the full expressions as well as the Mathematica code). The residue for pole at $y=H_{d} / \sqrt{x}$ is

$$
\begin{aligned}
- & x^{11} H_{d}^{13} z_{1}^{10}\left(z_{1}^{2}-z_{2}\right) z_{2}^{10}\left(z_{1} z_{2}-1\right)\left(z_{1}-z_{2}^{2}\right)\left[\left(x e_{1}-1\right)\left(x e_{2}-1\right)\left(x e_{3}-1\right)\left(H_{d}-x H_{u}\right)\right. \\
& \times\left(H_{d} H_{u}-1\right)\left(H_{d}-L_{1}\right)\left(H_{d} L_{1}-x\right)\left(H_{d}-L_{2}\right)\left(H_{d} L_{2}-x\right)\left(H_{d}-L_{3}\right)\left(H_{d} L_{3}-x\right)\left(\sqrt[3]{x} d_{1}-z_{1}\right) \\
& \times\left(\sqrt[3]{x} d_{2}-z_{1}\right)\left(\sqrt[3]{x} d_{3}-z_{1}\right)\left(x^{2 / 3} z_{1}-u_{1}\right)\left(x^{2 / 3} z_{1}-u_{2}\right)\left(x^{2 / 3} z_{1}-u_{3}\right)\left(H_{d}-x^{2 / 3} Q_{1} z_{1}\right) \\
& \times\left(H_{d} Q_{1} z_{1}-\sqrt[3]{x}\right)\left(H_{d}-x^{2 / 3} Q_{2} z_{1}\right)\left(H_{d} Q_{2} z_{1}-\sqrt[3]{x}\right)\left(H_{d}-x^{2 / 3} Q_{3} z_{1}\right)\left(H_{d} Q_{3} z_{1}-\sqrt[3]{x}\right) \\
& \times\left(\sqrt[3]{x} d_{1} z_{1}-z_{2}\right)\left(\sqrt[3]{x} d_{2} z_{1}-z_{2}\right)\left(\sqrt[3]{x} d_{3} z_{1}-z_{2}\right)\left(H_{d} Q_{1}-\sqrt[3]{x} z_{2}\right)\left(H_{d} Q_{2}-\sqrt[3]{x} z_{2}\right) \\
& \times\left(H_{d} Q_{3}-\sqrt[3]{x} z_{2}\right)\left(x^{2 / 3} z_{2}-u_{1} z_{1}\right)\left(x^{2 / 3} z_{2}-u_{2} z_{1}\right)\left(x^{2 / 3} z_{2}-u_{3} z_{1}\right)\left(\sqrt[3]{x} d_{1} z_{2}-1\right) \\
& \times\left(\sqrt[3]{x} d_{2} z_{2}-1\right)\left(\sqrt[3]{x} d_{3} z_{2}-1\right)\left(H_{d} z_{2}-x^{2 / 3} Q_{1}\right)\left(H_{d} z_{2}-x^{2 / 3} Q_{2}\right)\left(H_{d} z_{2}-x^{2 / 3} Q_{3}\right) \\
& \times\left(H_{d} z_{1}-x^{2 / 3} Q_{1} z_{2}\right)\left(H_{d} Q_{1} z_{2}-\sqrt[3]{x} z_{1}\right)\left(H_{d} z_{1}-x^{2 / 3} Q_{2} z_{2}\right)\left(H_{d} Q_{2} z_{2}-\sqrt[3]{x} z_{1}\right) \\
& \left.\times\left(H_{d} z_{1}-x^{2 / 3} Q_{3} z_{2}\right)\left(H_{d} Q_{3} z_{2}-\sqrt[3]{x} z_{1}\right)\left(x^{2 / 3}-u_{1} z_{2}\right)\left(x^{2 / 3}-u_{2} z_{2}\right)\left(x^{2 / 3}-u_{3} z_{2}\right)\right]^{-1},
\end{aligned}
$$

and the residue for pole at $y=L_{1} / \sqrt{x}$ is

$$
\begin{aligned}
x^{11} & L_{1}^{13} z_{1}^{10}\left(z_{1}^{2}-z_{2}\right) z_{2}^{10}\left(z_{1} z_{2}-1\right)\left(z_{1}-z_{2}^{2}\right)\left[\left(x e_{1}-1\right)\left(x e_{2}-1\right)\left(x e_{3}-1\right)\left(L_{1}-H_{d}\right)\right. \\
& \times\left(L_{1}-x H_{u}\right)\left(H_{d} L_{1}-x\right)\left(H_{u} L_{1}-1\right)\left(L_{1}-L_{2}\right)\left(L_{1} L_{2}-x\right)\left(L_{1}-L_{3}\right)\left(L_{1} L_{3}-x\right)\left(\sqrt[3]{x} d_{1}-z_{1}\right) \\
& \times\left(\sqrt[3]{x} d_{2}-z_{1}\right)\left(\sqrt[3]{x} d_{3}-z_{1}\right)\left(x^{2 / 3} z_{1}-u_{1}\right)\left(x^{2 / 3} z_{1}-u_{2}\right)\left(x^{2 / 3} z_{1}-u_{3}\right)\left(L_{1}-x^{2 / 3} Q_{1} z_{1}\right) \\
& \times\left(L_{1} Q_{1} z_{1}-\sqrt[3]{x}\right)\left(L_{1}-x^{2 / 3} Q_{2} z_{1}\right)\left(L_{1} Q_{2} z_{1}-\sqrt[3]{x}\right)\left(L_{1}-x^{2 / 3} Q_{3} z_{1}\right)\left(L_{1} Q_{3} z_{1}-\sqrt[3]{x}\right) \\
& \times\left(\sqrt[3]{x} d_{1} z_{1}-z_{2}\right)\left(\sqrt[3]{x} d_{2} z_{1}-z_{2}\right)\left(\sqrt[3]{x} d_{3} z_{1}-z_{2}\right)\left(L_{1} Q_{1}-\sqrt[3]{x} z_{2}\right)\left(L_{1} Q_{2}-\sqrt[3]{x} z_{2}\right) \\
& \times\left(L_{1} Q_{3}-\sqrt[3]{x} z_{2}\right)\left(x^{2 / 3} z_{2}-u_{1} z_{1}\right)\left(x^{2 / 3} z_{2}-u_{2} z_{1}\right)\left(x^{2 / 3} z_{2}-u_{3} z_{1}\right)\left(\sqrt[3]{x} d_{1} z_{2}-1\right) \\
& \times\left(\sqrt[3]{x} d_{2} z_{2}-1\right)\left(\sqrt[3]{x} d_{3} z_{2}-1\right)\left(L_{1} z_{2}-x^{2 / 3} Q_{1}\right)\left(L_{1} z_{2}-x^{2 / 3} Q_{2}\right)\left(L_{1} z_{2}-x^{2 / 3} Q_{3}\right) \\
& \times\left(L_{1} z_{1}-x^{2 / 3} Q_{1} z_{2}\right)\left(L_{1} Q_{1} z_{2}-\sqrt[3]{x} z_{1}\right)\left(L_{1} z_{1}-x^{2 / 3} Q_{2} z_{2}\right)\left(L_{1} Q_{2} z_{2}-\sqrt[3]{x} z_{1}\right) \\
& \left.\times\left(L_{1} z_{1}-x^{2 / 3} Q_{3} z_{2}\right)\left(L_{1} Q_{3} z_{2}-\sqrt[3]{x} z_{1}\right)\left(x^{2 / 3}-u_{1} z_{2}\right)\left(x^{2 / 3}-u_{2} z_{2}\right)\left(x^{2 / 3}-u_{3} z_{2}\right)\right]^{-1} .
\end{aligned}
$$


We can see from the above expressions that the denominators have over 40 terms and the current built-in functions from the likes of Mathematica have difficulties in finding the common denominator and summing over the numerators even for these 2 terms, let along summing over all 14. The reason is that with 40 terms, when brought to the same denominator and expanded, we are confronted with $2^{40} \sim$ $10^{12}$ monomial terms; factoring a polynomial with this many terms is clearly hopeless. It is remarkable that we could forge ahead and obtain a final answer, as we shall see.

\section{The $z_{1}$ integral}

To circumvent the issue of summing all 14 residues from the $y$ integral, we perform the contour integral separately for each of the 14 expressions. Specifically, these 14 rational expressions give poles whose positions are not fixed by the condition in (4.5) with respect to the contour $\left|z_{1}\right|=1$, i.e., we do not know whether some on the poles are inside or outside the contour. This simply means that the contour integral cannot be performed with the data available to us. However, the plethystic integral for the MSSM should lead to a definite result and this indeterminacy of pole positions of the intermediate steps should be a result of the redundancy of doing the 14 integrals separately and not combining them into one rational expression. If we could combine to previous 14 expressions into a single rational function with common factors canceled between top and bottom, the terms that give indeterminate pole positions in the denominators should disappear.

Therefore, we can simply make a choice for the fugacities. The ultimate answer cannot depend on this choice by construction. We take the following choice:

$$
\begin{array}{rlrl}
L_{1} & =\frac{49}{51}, & L_{2}=\frac{23}{34}, & L_{3}=\frac{25}{34}, \\
u_{1}=\frac{101}{102}, & u_{2}=\frac{91}{102}, & u_{3}=\frac{14}{17}, \\
e_{1}=\frac{2}{3}, & e_{2}=\frac{2}{51}, & e_{3}=\frac{11}{17},
\end{array}
$$

At first sight, this choice of variable seems random and arbitrary. Indeed, this choice is made randomly by Mathematica subject to the constraints that all the fugacities have modulus smaller than 1 . It is reasonable to argue that there are infinitely many such choices and one could possibly obtain some other final results with other choice of fugacities. However, the final answer from this choice of fugacities shows that the denominator is in Euler form of $\prod\left(1-t^{n}\right)^{a_{n}}$, the coefficients of numerators are all integer and the Taylor expansion of the Hilbert series that is a rational function, gives positive integer coefficients. It is reassuring to see all the checks for a legitimate Hilbert series go through and it also would be satisfying to see that different choices of fugacities can lead to the same result to appear in any future works.

With this choice of variables at hand, we can fully determine whether a pole is inside or outside the contour, thus we know whether the pole should be included when residues are collected. For example, we have a pole for the first of the 14 expression as $z_{1}=\frac{L_{2}}{Q_{1} x^{2 / 3}}$. If we only use the condition from Eq. (4.5), we will not be able to decide if this should be included in the residue or not. However, with the choice from (5.4), it is clear that this should be discarded since it is outside the contour of $\left|z_{1}\right|=1$.

Using this choice of fugacities, we arrive at a total of 198 poles that are inside the contour for collecting residues. We obtain the integral for each individual rational functions using Mathematica built-in functions. After performing the 198 integrals, we clean up the results to reduce the amount of work for later integrals. This is done by collecting the terms sharing the same denominator and combining them into a single term. After these procedures, we arrive at a total of 114 terms (again, available at the aforementioned URL) that need to be integrated separately.

\section{The $z_{2}$ integral}

Using the results from the previous step, we proceed to perform these 114 integrals over the variable $z_{2}$. Using the choice of fugacities (5.4), the number of poles found to be located inside the contour $\left|z_{2}\right|=1$ is 1622 . This amount of computation requires under 1 hour to complete on a laptop/PC with 4 cores using Mathematica built-in functions. However, there are large amount of redundancies within this computation. It can be seen that there are terms that simply cancel when we sum all the terms and the number of terms is reduced to 838. In addition, we can use the same method in the previous paragraph to collect together terms that share the same denominator. The number of terms is now reduced to 574 to enter our final integration over $x$, which is quite reasonable.

\section{The $x$ integral}

First, the number of poles that are inside contour $|x|=1$ with the fugacity choice (5.4) is 3106 . We proceed normally with the integral as before. To clean up the redundancies within these results, we sum up all the terms so that some of the terms will just cancel as they are simply 
negative of each other. In addition, terms sharing common denominator are collected. Finally, we obtain a list of 1538 terms. One important aspect of these results comes from the fact that even when we started with a plethystic integral with fractional power $1 / 6$ in variable $x$, we still end up with all terms having integer exponents and coefficients. However, the raw results of 1538 terms contain terms with fractional exponents in some variables. Remarkably, these fractional exponents combine into integer ones when summed up. Of course, the final answer for the Hilbert series is a rational function and cannot contain any fractional powers. These extraordinary cancellations give us confidence that we are indeed doing the right thing.

To get a flavor of these terms, we present two of these terms which combine to give integer exponents, viz.,

$$
\begin{aligned}
& Q_{3}^{11} u_{1}^{13} u_{2}^{7} u_{3}^{6}\left[2\left(Q_{3}-Q_{1}\right)\left(Q_{3}-Q_{2}\right)\left(u_{1}-u_{2}\right)\left(u_{1}-u_{3}\right)^{2}\left(u_{2}-u_{3}\right)\left(d_{1} \sqrt{u_{1}} \sqrt{u_{2}}-\sqrt{u_{3}}\right)\right. \\
& \quad \times\left(d_{2} \sqrt{u_{1}} \sqrt{u_{2}}-\sqrt{u_{3}}\right)\left(d_{3} \sqrt{u_{1}} \sqrt{u_{2}}-\sqrt{u_{3}}\right)\left(d_{1} \sqrt{u_{1}} \sqrt{u_{3}}-\sqrt{u_{2}}\right)\left(d_{2} \sqrt{u_{1}} \sqrt{u_{3}}-\sqrt{u_{2}}\right) \\
& \quad \times\left(d_{3} \sqrt{u_{1}} \sqrt{u_{3}}-\sqrt{u_{2}}\right)\left(\sqrt{u_{1}}-d_{1} \sqrt{u_{2}} \sqrt{u_{3}}\right)\left(\sqrt{u_{1}}-d_{2} \sqrt{u_{2}} \sqrt{u_{3}}\right)\left(\sqrt{u_{1}}-d_{3} \sqrt{u_{2}} \sqrt{u_{3}}\right) \\
& \quad \times\left(e_{1} \sqrt{u_{1}} \sqrt{u_{2}} \sqrt{u_{3}}-1\right)\left(e_{2} \sqrt{u_{1}} \sqrt{u_{2}} \sqrt{u_{3}}-1\right)\left(e_{3} \sqrt{u_{1}} \sqrt{u_{2}} \sqrt{u_{3}}-1\right)\left(Q_{3} u_{1}-Q_{1} u_{2}\right)\left(Q_{3} u_{1}-Q_{2} u_{2}\right) \\
& \quad \times\left(Q_{1} Q_{3} \sqrt{u_{1}} \sqrt{u_{2}}-\sqrt{u_{3}}\right)\left(Q_{2} Q_{3} \sqrt{u_{1}} \sqrt{u_{2}}-\sqrt{u_{3}}\right)\left(Q_{3}^{2} \sqrt{u_{1}} \sqrt{u_{2}}-\sqrt{u_{3}}\right)\left(Q_{1} Q_{3} \sqrt{u_{1}} \sqrt{u_{3}}-\sqrt{u_{2}}\right) \\
& \quad \times\left(Q_{2} Q_{3} \sqrt{u_{1}} \sqrt{u_{3}}-\sqrt{u_{2}}\right)\left(Q_{3}^{2} \sqrt{u_{1}} \sqrt{u_{3}}-\sqrt{u_{2}}\right)\left(Q_{1} Q_{3} u_{1}^{3 / 2}-\sqrt{u_{2}} \sqrt{u_{3}}\right)\left(Q_{2} Q_{3} u_{1}^{3 / 2}-\sqrt{u_{2}} \sqrt{u_{3}}\right) \\
& \quad \times\left(Q_{3} u_{1}-Q_{1} u_{3}\right)\left(Q_{3} u_{1}-Q_{2} u_{3}\right)\left(Q_{3} u_{1}-H_{d}\right)\left(H_{d} Q_{3} \sqrt{u_{1}}-\sqrt{u_{2}} \sqrt{u_{3}}\right)\left(H_{u} Q_{3} u_{1}-1\right) \\
& \quad \times\left(Q_{3} \sqrt{u_{1}}-H_{u} \sqrt{u_{2}} \sqrt{u_{3}}\right)\left(Q_{3} u_{1}-L_{1}\right)\left(Q_{3} u_{1}-L_{2}\right)\left(Q_{3} u_{1}-L_{3}\right) \\
& \left.\quad \times\left(L_{1} Q_{3} \sqrt{u_{1}}-\sqrt{u_{2}} \sqrt{u_{3}}\right)\left(L_{2} Q_{3} \sqrt{u_{1}}-\sqrt{u_{2}} \sqrt{u_{3}}\right)\left(L_{3} Q_{3} \sqrt{u_{1}}-\sqrt{u_{2}} \sqrt{u_{3}}\right)\right]^{-1},
\end{aligned}
$$

and

$$
\begin{aligned}
& Q_{3}^{11} u_{1}^{13} u_{2}^{7} u_{3}^{6}\left[2\left(Q_{3}-Q_{1}\right)\left(Q_{3}-Q_{2}\right)\left(u_{1}-u_{2}\right)\left(u_{1}-u_{3}\right)^{2}\left(u_{2}-u_{3}\right)\left(d_{1} \sqrt{u_{1}} \sqrt{u_{2}}+\sqrt{u_{3}}\right)\right. \\
& \quad \times\left(d_{2} \sqrt{u_{1}} \sqrt{u_{2}}+\sqrt{u_{3}}\right)\left(d_{3} \sqrt{u_{1}} \sqrt{u_{2}}+\sqrt{u_{3}}\right)\left(d_{1} \sqrt{u_{1}} \sqrt{u_{3}}+\sqrt{u_{2}}\right)\left(d_{2} \sqrt{u_{1}} \sqrt{u_{3}}+\sqrt{u_{2}}\right) \\
& \quad \times\left(d_{3} \sqrt{u_{1}} \sqrt{u_{3}}+\sqrt{u_{2}}\right)\left(d_{1} \sqrt{u_{2}} \sqrt{u_{3}}+\sqrt{u_{1}}\right)\left(d_{2} \sqrt{u_{2}} \sqrt{u_{3}}+\sqrt{u_{1}}\right)\left(d_{3} \sqrt{u_{2}} \sqrt{u_{3}}+\sqrt{u_{1}}\right) \\
& \quad \times\left(e_{1} \sqrt{u_{1}} \sqrt{u_{2}} \sqrt{u_{3}}+1\right)\left(e_{2} \sqrt{u_{1}} \sqrt{u_{2}} \sqrt{u_{3}}+1\right)\left(e_{3} \sqrt{u_{1}} \sqrt{u_{2}} \sqrt{u_{3}}+1\right)\left(Q_{3} u_{1}-Q_{1} u_{2}\right) \\
& \quad \times\left(Q_{3} u_{1}-Q_{2} u_{2}\right)\left(Q_{1} Q_{3} \sqrt{u_{1}} \sqrt{u_{2}}+\sqrt{u_{3}}\right)\left(Q_{2} Q_{3} \sqrt{u_{1}} \sqrt{u_{2}}+\sqrt{u_{3}}\right)\left(Q_{3}^{2} \sqrt{u_{1}} \sqrt{u_{2}}+\sqrt{u_{3}}\right) \\
& \quad \times\left(Q_{1} Q_{3} \sqrt{u_{1}} \sqrt{u_{3}}+\sqrt{u_{2}}\right)\left(Q_{2} Q_{3} \sqrt{u_{1}} \sqrt{u_{3}}+\sqrt{u_{2}}\right)\left(Q_{3}^{2} \sqrt{u_{1}} \sqrt{u_{3}}+\sqrt{u_{2}}\right) \\
& \quad \times\left(Q_{1} Q_{3} u_{1}^{3 / 2}+\sqrt{u_{2}} \sqrt{u_{3}}\right)\left(Q_{2} Q_{3} u_{1}^{3 / 2}+\sqrt{u_{2}} \sqrt{u_{3}}\right)\left(Q_{3} u_{1}-Q_{1} u_{3}\right)\left(Q_{3} u_{1}-Q_{2} u_{3}\right) \\
& \quad \times\left(Q_{3} u_{1}-H_{d}\right)\left(H_{d} Q_{3} \sqrt{u_{1}}+\sqrt{u_{2}} \sqrt{u_{3}}\right)\left(H_{u} Q_{3} u_{1}-1\right)\left(H_{u} \sqrt{u_{2}} \sqrt{u_{3}}+Q_{3} \sqrt{u_{1}}\right) \\
& \quad \times\left(Q_{3} u_{1}-L_{1}\right)\left(Q_{3} u_{1}-L_{2}\right)\left(Q_{3} u_{1}-L_{3}\right)\left(L_{1} Q_{3} \sqrt{u_{1}}+\sqrt{u_{2}} \sqrt{u_{3}}\right)\left(L_{2} Q_{3} \sqrt{u_{1}}+\sqrt{u_{2}} \sqrt{u_{3}}\right) \\
& \left.\quad \times\left(L_{3} Q_{3} \sqrt{u_{1}}+\sqrt{u_{2}} \sqrt{u_{3}}\right)\right]^{-1} .
\end{aligned}
$$

As one can see, both expressions are sprinkled with troubling terms involving $\sqrt{u_{i}}$ and $u_{i}^{3 / 2}$. Summing the expression (5.5) and (5.6) gives the common denominator to be

$$
\begin{aligned}
& 2\left(Q_{3}-Q_{1}\right)\left(Q_{3}-Q_{2}\right)\left(u_{1}-u_{2}\right)\left(u_{1}-u_{3}\right)^{2}\left(u_{2}-u_{3}\right)\left(d_{1}^{2} u_{1} u_{2}-u_{3}\right)\left(d_{2}^{2} u_{1} u_{2}-u_{3}\right)\left(d_{3}^{2} u_{1} u_{2}-u_{3}\right) \\
& \quad \times\left(d_{1}^{2} u_{1} u_{3}-u_{2}\right)\left(d_{2}^{2} u_{1} u_{3}-u_{2}\right)\left(d_{3}^{2} u_{1} u_{3}-u_{2}\right)\left(u_{1}-d_{1}^{2} u_{2} u_{3}\right)\left(u_{1}-d_{2}^{2} u_{2} u_{3}\right)\left(u_{1}-d_{3}^{2} u_{2} u_{3}\right) \\
& \quad \times\left(e_{1}^{2} u_{1} u_{2} u_{3}-1\right)\left(e_{2}^{2} u_{1} u_{2} u_{3}-1\right)\left(e_{3}^{2} u_{1} u_{2} u_{3}-1\right)\left(Q_{3} u_{1}-Q_{1} u_{2}\right)\left(Q_{3} u_{1}-Q_{2} u_{2}\right)\left(Q_{1}^{2} Q_{3}^{2} u_{1} u_{2}-u_{3}\right) \\
& \quad \times\left(Q_{2}^{2} Q_{3}^{2} u_{1} u_{2}-u_{3}\right)\left(Q_{3}^{4} u_{1} u_{2}-u_{3}\right)\left(Q_{3} u_{1}-Q_{1} u_{3}\right)\left(Q_{3} u_{1}-Q_{2} u_{3}\right)\left(Q_{1}^{2} Q_{3}^{2} u_{1} u_{3}-u_{2}\right) \\
& \quad \times\left(Q_{2}^{2} Q_{3}^{2} u_{1} u_{3}-u_{2}\right)\left(Q_{3}^{4} u_{1} u_{3}-u_{2}\right)\left(Q_{1}^{2} Q_{3}^{2} u_{1}^{3}-u_{2} u_{3}\right)\left(Q_{2}^{2} Q_{3}^{2} u_{1}^{3}-u_{2} u_{3}\right)\left(Q_{3} u_{1}-H_{d}\right) \\
& \quad \times\left(H_{d}^{2} Q_{3}^{2} u_{1}-u_{2} u_{3}\right)\left(H_{u} Q_{3} u_{1}-1\right)\left(Q_{3}^{2} u_{1}-H_{u}^{2} u_{2} u_{3}\right)\left(Q_{3} u_{1}-L_{1}\right)\left(Q_{3} u_{1}-L_{2}\right) \\
& \quad \times\left(Q_{3} u_{1}-L_{3}\right)\left(L_{1}^{2} Q_{3}^{2} u_{1}-u_{2} u_{3}\right)\left(L_{2}^{2} Q_{3}^{2} u_{1}-u_{2} u_{3}\right)\left(L_{3}^{2} Q_{3}^{2} u_{1}-u_{2} u_{3}\right)
\end{aligned}
$$


and all fractional powers disappear. It is indeed reassuring that of the 3106 terms, any term with a fractional power therein has exactly 1 partner which cancels it upon summation. This is guaranteed by representation theory (characters) in the Molien-Weyl formula. The numerator is expanded to check for integer coefficients and exponents (note that this expansion gives us over 4 millions terms). The integer criterion indeed checks out for this example and the first few terms are

$$
\begin{aligned}
& 2 d_{1} d_{2} d_{3} H_{d} L_{1} L_{2} L_{3} Q_{1}^{2} Q_{2}^{2} Q_{3}^{22} u_{2}^{13} u_{3}^{6} u_{1}^{23}+2 d_{1} d_{2} H_{d} L_{1} L_{2} L_{3} Q_{1}^{2} Q_{2} Q_{3}^{21} u_{2}^{12} u_{3}^{7} u_{1}^{22} \\
& \quad+2 d_{1} d_{3} H_{d} L_{1} L_{2} L_{3} Q_{1}^{2} Q_{2} Q_{3}^{21} u_{2}^{12} u_{3}^{7} u_{1}^{22}+2 d_{2} d_{3} H_{d} L_{1} L_{2} L_{3} Q_{1}^{2} Q_{2} Q_{3}^{21} u_{2}^{12} u_{3}^{7} u_{1}^{22} \\
& \quad+2 d_{1} d_{2} H_{d} L_{1} L_{2} L_{3} Q_{1}^{2} Q_{2}^{2} Q_{3}^{20} u_{2}^{12} u_{3}^{7} u_{1}^{22}+2 d_{1} d_{3} H_{d} L_{1} L_{2} L_{3} Q_{1}^{2} Q_{2}^{2} Q_{3}^{20} u_{2}^{12} u_{3}^{7} u_{1}^{22} \\
& \quad+2 d_{2} d_{3} H_{d} L_{1} L_{2} L_{3} Q_{1}^{2} Q_{2}^{2} Q_{3}^{20} u_{2}^{12} u_{3}^{7} u_{1}^{22}+2 d_{1} d_{2} d_{3} H_{d} L_{1} L_{2} L_{3} Q_{1} Q_{2} Q_{3}^{20} u_{2}^{12} u_{3}^{7} u_{1}^{22} \\
& \quad+2 d_{1} d_{2} d_{3} H_{d} L_{1} L_{2} L_{3} Q_{1} Q_{2}^{2} Q_{3}^{19} u_{2}^{12} u_{3}^{7} u_{1}^{22}+2 d_{1} d_{2} d_{3} H_{d} L_{1} L_{2} L_{3} Q_{1}^{2} Q_{2} Q_{3}^{19} u_{2}^{12} u_{3}^{7} u_{1}^{22}+\ldots
\end{aligned}
$$

\section{B. Unrefining the Hilbert series}

After the previous section, we now have a list of 1538 rational expressions that should be combined into a single rational function, which is the Hilbert series for the MSSM. However, due to the complexity of each rational expression, it is impossible to combine even two terms under a common denominator using common computer packages such as Mathematica or Mccaulay2. To show the complexity of each term, we present an example below

$$
\begin{aligned}
H_{d}^{15} & Q_{1}^{14} u_{1}^{9}\left[\left(Q_{1}-Q_{2}\right)^{2}\left(Q_{1}-Q_{3}\right)^{2}\left(u_{1}-u_{2}\right)^{2}\left(u_{1}-u_{3}\right)^{2}\left(H_{d} H_{u}-1\right)\right. \\
& \times\left(H_{d}-L_{1}\right)\left(H_{d}-L_{2}\right)\left(H_{d}-L_{3}\right)\left(H_{d} Q_{1}^{3}-1\right)\left(H_{d} Q_{1}^{2} Q_{2}-1\right)\left(H_{d} Q_{1}^{2} Q_{3}-1\right) \\
& \times\left(d_{1} H_{d} Q_{1}-1\right)^{2}\left(d_{2} H_{d} Q_{1}-1\right)^{2}\left(d_{3} H_{d} Q_{1}-1\right)^{2}\left(H_{d}^{2} Q_{1} Q_{2}-u_{1}\right) \\
& \times\left(H_{d}^{2} Q_{1} Q_{3}-u_{1}\right)\left(H_{d}-Q_{1} u_{1}\right)\left(H_{d}-Q_{2} u_{1}\right)^{2}\left(H_{d}-Q_{3} u_{1}\right)^{2} \\
& \times\left(H_{d}^{2} Q_{1}^{2}-u_{2}\right)\left(H_{d}^{2} Q_{1}^{2}-u_{3}\right)\left(H_{u} Q_{1} u_{1}-1\right)\left(L_{1}-Q_{1} u_{1}\right)\left(L_{2}-Q_{1} u_{1}\right) \\
& \times\left(L_{3}-Q_{1} u_{1}\right)\left(H_{d} Q_{1}-d_{1} u_{1}\right)\left(H_{d} Q_{1}-d_{2} u_{1}\right)\left(H_{d} Q_{1}-d_{3} u_{1}\right)\left(e_{1} H_{d} Q_{1} u_{1}-1\right) \\
& \left.\times\left(e_{2} H_{d} Q_{1} u_{1}-1\right)\left(e_{3} H_{d} Q_{1} u_{1}-1\right)\right]^{-1} \\
L_{1}^{15} & Q_{1}^{14} u_{1}^{9}\left[\left(L_{1}-L_{2}\right)\left(L_{1}-L_{3}\right)\left(Q_{1}-Q_{2}\right)^{2}\left(Q_{1}-Q_{3}\right)^{2}\left(u_{1}-u_{2}\right)^{2}\left(u_{1}-u_{3}\right)^{2}\right. \\
& \times\left(L_{1}-H_{d}\right)\left(H_{u} L_{1}-1\right)\left(L_{1} Q_{1}^{3}-1\right)\left(L_{1} Q_{1}^{2} Q_{2}-1\right)\left(L_{1} Q_{1}^{2} Q_{3}-1\right)\left(d_{1} L_{1} Q_{1}-1\right)^{2} \\
& \times\left(d_{2} L_{1} Q_{1}-1\right)^{2}\left(d_{3} L_{1} Q_{1}-1\right)^{2}\left(H_{d}-Q_{1} u_{1}\right)\left(H_{u} Q_{1} u_{1}-1\right)\left(L_{1}^{2} Q_{1} Q_{2}-u_{1}\right) \\
& \times\left(L_{1}^{2} Q_{1} Q_{3}-u_{1}\right)\left(L_{1}-Q_{1} u_{1}\right)\left(L_{2}-Q_{1} u_{1}\right)\left(L_{3}-Q_{1} u_{1}\right)\left(L_{1}-Q_{2} u_{1}\right)^{2}\left(L_{1}-Q_{3} u_{1}\right)^{2} \\
& \times\left(L_{1}^{2} Q_{1}^{2}-u_{2}\right)\left(L_{1}^{2} Q_{1}^{2}-u_{3}\right)\left(L_{1} Q_{1}-d_{1} u_{1}\right)\left(L_{1} Q_{1}-d_{2} u_{1}\right)\left(L_{1} Q_{1}-d_{3} u_{1}\right) \\
& \left.\times\left(e_{1} L_{1} Q_{1} u_{1}-1\right)\left(e_{2} L_{1} Q_{1} u_{1}-1\right)\left(e_{3} L_{1} Q_{1} u_{1}-1\right)\right]^{-1} .
\end{aligned}
$$

As we can see, a typical rational expression has roughly 33 factors in the denominator, thus combining them implies finding the lowest common multiple between each denominator with roughly 30 factors. That is, we need to compute resultants between all pairs from 1538 multivariate polynomials each with about $2^{30} \simeq 10^{9}$ monomial terms, rendering the process impractical on an average $\mathrm{PC} /$ Laptop. Nevertheless, each of the 1538 rational functions, as seen from the above expression, is not too complicated. Therefore, we have

PROPOSITION 5.1: The multivariate, fully refined Hilbert series for the MSSM (without superpotential) is a 
TABLE V. The weights for unrefining the Hilbert series of MSSM.

\begin{tabular}{ccccccccccccccccc}
\hline \hline$d_{1}$ & $d_{2}$ & $d_{3}$ & $e_{1}$ & $e_{2}$ & $e_{3}$ & $H_{d}$ & $H_{u}$ & $L_{1}$ & $L_{2}$ & $L_{3}$ & $Q_{1}$ & $Q_{2}$ & $Q_{3}$ & $u_{1}$ & $u_{2}$ & $u_{3}$ \\
$t^{512}$ & $t^{256}$ & $t^{128}$ & $t^{512}$ & $t^{512}$ & $t^{512}$ & $t^{512}$ & $t^{512}$ & $t^{256}$ & $t^{128}$ & $t^{64}$ & $t^{32}$ & $t^{16}$ & $t^{8}$ & $t^{4}$ & $t^{2}$ & $t$ \\
\hline \hline
\end{tabular}

sum of 1538 rational functions in 17 variables, viz., $Q_{i}, L_{i}$, $u_{i}, d_{i}, e_{i}$ for $i=1,2,3$ as well as $H_{u}$ and $H_{d}$. The full expressions are given in this link.

It is difficult to extract geometrical information directly from this full Hilbert series. Happily, we can "unrefine," i.e., force the Hilbert series to be univariate by setting all 18 variables to a single one, say $t$, but to different powers. That is, the unrefinement is simply a substitution of variables by expression $t^{\alpha}$, where $t$ is also a fugacity and $\alpha$ is the weight for particular variable that is being substituted. This weight normally corresponds to some particular $U(1)$ charges and some particular choice should render the common denominator nonzero when unrefining (cf. [13]). This particular choice of weight we make is as follows:

With this choice of weights, we are able to greatly simplify the expression in Proposition 5.1 to a single rational function with denominator being in Euler product form and numerator having integer coefficients and exponents, as we shall now see. The astute reader might argue that this choice of weights seems arbitrary and that we can as well make other choices with more straightforward physical implications. In fact, we have made weight choices that represent baryron numbers, lepton numbers etc and find that they all give 0 in the denominator, thus disqualifying themselves as legitimate weight choices. This particular choice is by far the most reasonable weights that do not give 0 in the denominator in the rational function of Hilbert series.

\section{Simplifying the unrefined Hilbert series}

After the unrefinement with weights in Table V, the Hilbert series is simplified into a rational function, with a polynomial of degree 816,890 as the numerator and a denominator with 994 factors of total degree 824,397. Note that the factors are already in Euler form and should correspond to the GIOs which parametrize the VMS of the MSSM. Even this rational function looks unmanageable at first sight, we can still extract useful information out of it. Importantly, as a preliminary step, we need to perform a Taylor series in $t$ for the Hilbert series in order to know how many independent generators there are in each degree. In terms of the supersymmetric gauge theory, this counts the number of independent 1/2-BPS single-trace operators at each $U(1)$ charge [12,13]. Doing so we obtain:

$$
\begin{aligned}
H(t)= & 1+2 t^{2}+4 t^{3}+6 t^{4}+10 t^{5}+16 t^{6}+20 t^{7}+28 t^{8} \\
& +38 t^{9}+48 t^{10}+64 t^{11}+84 t^{12}+104 t^{13}+134 t^{14} \\
& +168 t^{15}+202 t^{16}+250 t^{17}+304 t^{18}+360 t^{19} \\
& +436 t^{20}+\mathcal{O}\left(t^{21}\right) .
\end{aligned}
$$

It is very assuring that all coefficients are non-negative, as it is a requirement in the series development of the Hilbert series (since it counts the number of independent monomials in the polynomial ring corresponding to the variety). This requires highly nontrivial conspiracy between the numerator and denominator since each contains many terms with explicitly negative coefficients. Furthermore, the leading term is 1 , as is also required. The coefficient list $\{1,0,2,4,6,10,16,20,28,38,48,64,84,104,134,168$, $202,250,304,360,436 \ldots\}$, unfortunately does not resemble anything known in the literature. It would be interesting indeed if this appeared in some combinatorial context.

It is important that we cover the details for simplifying the Hilbert series $H(t)=P(t) / Q(t)$ to a usable form. Now, since after adding up the various partial fractions from the Molien-Weyl integral, the numerator $P(t)$ is a polynomial of degree $816,890=2 \cdot 5 \cdot 81,689$ of no particularly apparent structure and the denominator $Q(t)$, one of degree $824,397=3 \cdot 7 \cdot 37 \cdot 1061$, it is already beyond conventional packages such as Mathematica to simplify it in any way. At least the denominator is already in Euler form ${ }^{4}$ consisting of 991 unique factors, ranging from 2 at degree 1 to 1 at degree 1664 in our weighting. ${ }^{5}$

We can present the full expression for the denominator in a compact form: by the array $w_{1}^{a_{1}}, w_{2}^{a_{2}}, \ldots$ we mean the polynomial $\left(1-t^{w_{1}}\right)^{a_{1}} \cdot\left(1-t^{w_{2}}\right)^{a_{2}} \ldots$. In this notation, the denominator $Q(t)$ is given by

\footnotetext{
${ }^{4}$ As a technical aside, we have to ensure that there are no terms like $\left(1+t^{w}\right)$ in the product in the denominator since the Euler form must have all terms strictly with the minus sign. We can guarantee this by multiplying, each time a term such as $\left(1+t^{w}\right)$ appear, numerator and denominator by $\left(1-t^{w}\right)$ so as to contribute a legitimate $\left(1-t^{2 w}\right)$ factor in the denominator. This actually happens only thrice: $\left(1+t^{8}\right)\left(1+t^{16}\right)\left(1+t^{24}\right)$ in our case.

${ }^{5}$ With this 991 we are in fact familiar. There are 991 generators of gauge invariants to the MSSM [6,7] However, by more recent recalculations, this number is slightly higher than the correct value [10]. But we shall see that after more simplification, the number of factors in the denominator reduces to 445 . Thus, at this stage, it seems to be a curious coincidence.
} 
$240282358376 \quad 386 \quad 392 \quad 400 \quad 408^{2} \quad 413 \quad 417422 \quad 423 \quad 424 \quad 427 \quad 429432^{2} \quad 433 \quad 434 \quad 435437$

$\begin{array}{llllllllllllllllllll}438 & 441 & 442 & 443 & 444 & 445 & 448 & 461 & 462 & 463 & 464^{2} & 465 & 466 & 469 & 470 & 471 & 472^{2} & 473 & 474 & 475\end{array}$

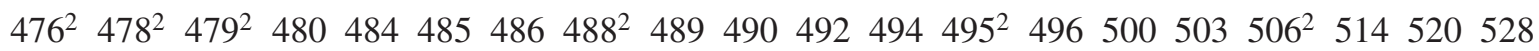

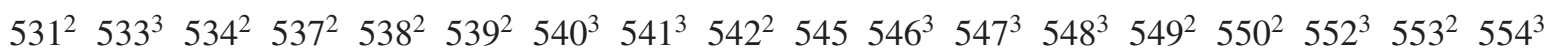

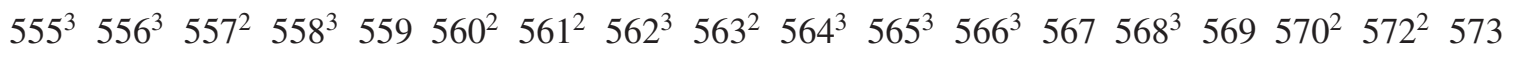

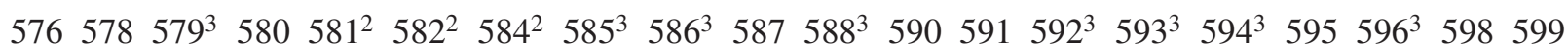

$\begin{array}{llllllllllllllllllll}600^{3} & 601 & 602^{3} & 604^{3} & 605 & 606 & 607 & 608^{2} & 609^{3} & 610^{3} & 611 & 612^{3} & 614 & 615 & 616^{3} & 620 & 623 & 624^{2} & 625 & 628\end{array}$

$\begin{array}{llllllllllllllllllll}631 & 632^{2} & 633 & 637 & 642 & 643^{3} & 644 & 645^{2} & 646^{2} & 648^{2} & 649^{3} & 650^{3} & 651 & 654 & 655 & 656^{3} & 657^{3} & 658^{3} & 659 & 662\end{array}$

$663664668669670671 \quad 672^{2} \quad 673^{3} \quad 674^{3} \quad 675 \quad 676^{3} 678679684686687688696700702703$

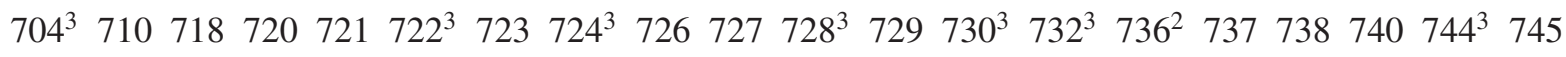

$\begin{array}{lllllllllllllllllllll}746^{3} & 747 & 748^{3} & 749 & 750 & 752^{3} & 753 & 758 & 760^{3} & 766 & 767 & 768 & 769 & 770 & 771^{3} & 772 & 773^{3} & 774^{2} & 776 & 777^{3}\end{array}$

$\begin{array}{llllllllllllllllllllll}778^{3} & 780^{3} & 781 & 782 & 784^{3} & 785^{3} & 786^{3} & 788^{3} & 790 & 792^{3} & 794 & 796 & 797 & 798 & 799 & 800^{2} & 801^{3} & 802^{3} & 803 & 804^{3}\end{array}$

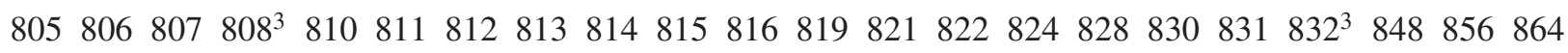

$872880888892894895896^{3} 903912920934936959963965974977978^{3} 980^{3} 982984^{3}$

$985986988991992994995996997999 \quad 1000^{3} 100110021004100710081011101210131014$

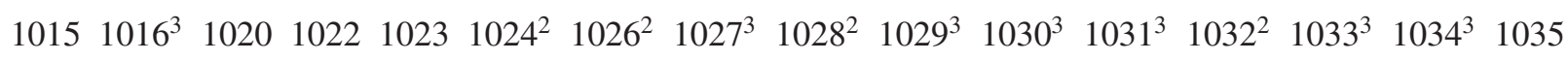

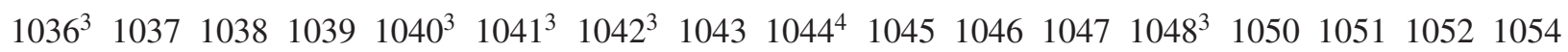

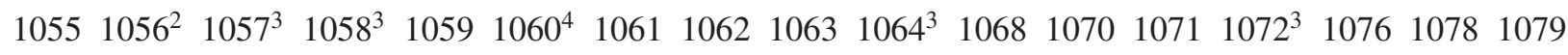

$1083108410861087 \quad 1088^{3} \quad 10901098 \quad 1100110111021103 \quad 1114112011241126112711361140$

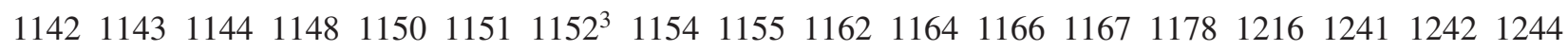

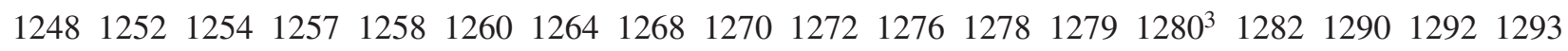

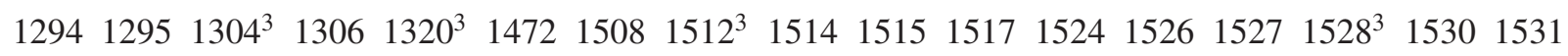

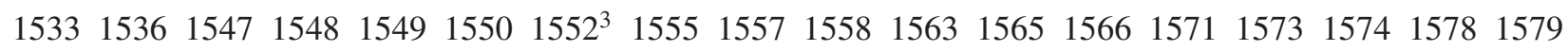

158115821587158915901664

We now need to extract as much of the list of factors in $Q(t)$ from the numerator $P(t)$. First, we know this is going to be possible because we can check that $P(1)=0$ (even on Mathematica this is still doable as this is simply the sum over all coefficients) so that it must divide $(1-t)$ at least once (and as we will see, many times). To efficiently perform factorization, we will use a so-called extended synthetic division algorithm [52] for monovariate polynomials. Luckily, there is an available Python/Sage implementation(c.f. Appendix B for a detailed discussion of this algorithm) of whose liberal use we will take advantage.
Our strategy is to first go over the 991 factors (with multiplicity this amounts to 1477 factors) of the form $1-t^{a}$ and try synthetic division into the numerator, this will cancel any such Euler factors therefrom. Doing so (and even with Python, it still takes on the order of 3 days on a regular laptop due to the large degree of the dividend), we find that the numerator now reduces to a polynomial $P_{1}(t)$, of degree $816,890-259,498=557,392$, (significantly reduced from the 816,890 of $P(t)$ ), likewise, the denominator reduces to $Q_{1}(t) \mathrm{m}$ with only 445 unique factors (and with multiplicity, 684 factors), in the shorthand notation for the Euler form, $Q_{1}(t)$ is 


\begin{abstract}
$\begin{array}{llllllllllllllllllll}240 & 282 & 358 & 376 & 386 & 392 & 400 & 408^{2} & 413 & 417 & 422 & 423 & 424 & 427 & 429 & 432^{2} & 433 & 434 & 435 & 437\end{array}$
$\begin{array}{llllllllllllllllllll}438 & 441 & 442 & 443 & 444 & 445 & 448 & 461 & 462 & 463 & 464^{2} & 465 & 466 & 469 & 470 & 471 & 472^{2} & 473 & 474 & 475\end{array}$

$\begin{array}{llllllllllllllllllll}476^{2} & 478^{2} & 479^{2} & 480 & 484 & 485 & 486 & 488^{2} & 489 & 490 & 492 & 494 & 495^{2} & 496 & 500 & 503 & 506^{2} & 514 & 520 & 528\end{array}$

$\begin{array}{llllllllllllllllllllllll}531^{2} & 533^{3} & 534^{2} & 537^{2} & 538^{2} & 539^{2} & 540^{3} & 541^{3} & 542^{2} & 545 & 546^{3} & 547^{3} & 548^{3} & 549^{2} & 550^{2} & 552^{3} & 553^{2} & 554^{3} & 555^{3} & 556^{3}\end{array}$

$\begin{array}{llllllllllllllllllll}57^{2} & 558^{3} & 559 & 560^{2} & 561^{2} & 562^{3} & 563^{2} & 564^{3} & 565^{3} & 566^{3} & 567 & 568^{3} & 569 & 570^{2} & 572^{2} & 573 & 576 & 578 & 579^{3} & 580\end{array}$

$\begin{array}{llllllllllllllllllll}581^{2} & 582^{2} & 584^{2} & 585^{3} & 586^{3} & 587 & 588^{3} & 590 & 591 & 592^{3} & 593^{3} & 594^{3} & 595 & 596^{3} & 598 & 599 & 600^{3} & 601 & 602^{3} & 604^{3}\end{array}$

$\begin{array}{llllllllllllllllllll}605 & 606 & 607 & 608^{2} & 609^{3} & 610^{3} & 611 & 612^{3} & 614 & 615 & 616^{3} & 620 & 623 & 624^{2} & 625 & 628 & 631 & 632^{2} & 633 & 637\end{array}$

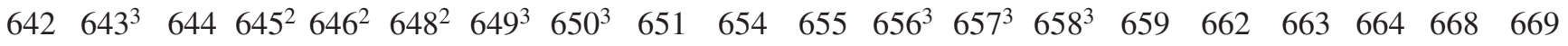

$\begin{array}{llllllllllllllllllll}670 & 671 & 672^{2} & 673^{3} & 674^{3} & 675 & 676^{3} & 678 & 679 & 684 & 686 & 687 & 688 & 696 & 700 & 702 & 703 & 704^{3} & 710 & 718\end{array}$

$\begin{array}{llllllllllllllllllll}720 & 721 & 722^{3} & 723 & 724^{3} & 726 & 727 & 728^{3} & 729 & 730^{3} & 732^{3} & 736^{2} & 737 & 738 & 740 & 744^{3} & 745 & 746^{3} & 747 & 748^{3}\end{array}$

$\begin{array}{lllllllllllllllllllll}749 & 750 & 752^{3} & 753 & 758 & 760^{3} & 766 & 767 & 768 & 769 & 770 & 771^{3} & 772 & 773^{3} & 774^{2} & 776 & 777^{3} & 778^{3} & 780^{3} & 781\end{array}$

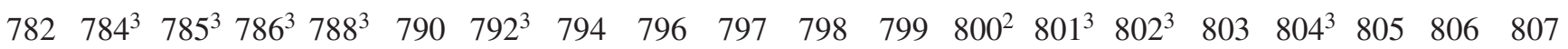

$\begin{array}{llllllllllllllllllll}808^{3} & 810 & 811 & 812 & 813 & 814 & 815 & 816 & 819 & 821 & 822 & 824 & 828 & 830 & 831 & 832^{3} & 848 & 856 & 864 & 872\end{array}$

$\begin{array}{llllllllllllllllllll}880 & 888 & 892 & 894 & 895 & 896^{3} & 903 & 912 & 920 & 934 & 936 & 959 & 963 & 965 & 974 & 977 & 978^{3} & 980^{3} & 982 & 984^{3}\end{array}$

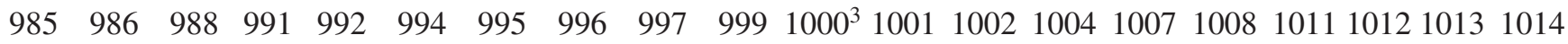

$10151016^{3} 1020102210231024^{2} 1026^{2} 1027^{3} 1028^{2} 1029^{3} 1030^{3} 1031^{3} 1032^{2} 1033^{3} 1034^{3} 10351036^{3} 103710381039$

$1040^{3} 1041^{3} 1042^{3} 10431044^{4} 1045104610471048^{3} 105010511052105410551056^{2} 1057^{3} 1058^{3} 10591060^{4} 1061$

$106210631064^{3} 1068107010711072^{3} 10761078107910831084108610871088^{3} 10901098110011011102$

$110311141120112411261127113611401142114311441148115011511152^{3} 11541155116211641166$

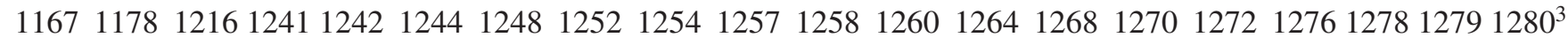

$1282129012921293129412951304^{3} 13061320^{3} 147215081512^{3} 1514151515171524152615271528^{3} 1530$

$15311533153615471548154915501552^{3} 155515571558156315651566157115731574157815791581$

15821587158915901664
\end{abstract}

It is also worthwhile to show a few terms of the numerator after this simplification

$$
\begin{aligned}
1 & +644 t+207692 t^{2}+44723872 t^{3}+7234295277 t^{4}+937600779818 t^{5}+101422033650142 t^{6} \\
& +9418304332530212 t^{7}+766466167260384451 t^{8}+55530492150394701928 t^{9} \\
& +3626455298524160306256 t^{10}+215630005089109137483320 t^{11}+11771050533831821348659441 t^{12} \\
& +\cdots+102352219991766 t^{556741}+944789516589 t^{556742}+7278808875 t^{556743}+44930916 t^{556744} \\
& +208335 t^{556745}+645 t^{556746}+t^{556747}
\end{aligned}
$$

Using the simplified set of numerator and denominator, we should proceed to extract more information such as dimension and degree of the variety. To do this, let us recall that there are two forms of Hilbert series in Eq. (2.2). However, our HS has a different form in the denominator due to our specific choice of weights. We can still obtain the dimension using the data at our disposal. The process is as follows

(1) Collect all the $(1-t)$ factors from the numerator, which in our case, the multiplicity is 644 .
(2) Count the number of Euler factors of the form $\left(1-t^{m_{i}}\right)$ with multiplicity, which is 684 . The dimension is simply $688-644=40$.

(3) To get the degree, we put the HS into the form

$$
\mathrm{HS}=\frac{(1-t)^{644} P_{1}(t)}{Q_{1}(t)},
$$

where $Q(t)$ is in Euler form and $P_{1}(1) \neq 0$. Now the degree is $P_{1}(1)$ with factors of 2 being pulled out 
and ignored as they come from our choice of weights. This is a rather large number about $-2.24 \times 10^{1633}$ and we present it in Sec. I.

Summarizing what we have obtained so far, we start with the plethystic integral proposition 4.1 and perform the contour integral stepwise by finding residues of the integrand. Due to the complexities of combining the intermediate results under the common denominator, we perform the ensuing integrations separately as discussed after Eq. (5.3). This brings extra redundancies during the integration as it is not possible to determine whether some poles should be included in the integration since we do not know if they are inside the contour or not. To eliminate these redundancies, we make some choice of fugacities in Eq. (5.4) and this choice is justified by the final result which has Euler form in its denominator and is free from fractional powers and coefficients. After obtaining the final unrefined results, we are still presented with the problem of extracting useful geometric information out of this complex expression. Unrefining the expression as suggested in Table $\mathrm{V}$ is thus necessary. Carrying out this procedure, we still have to use the extended synthetic division algorithm in Appendix B to further simplify the rational function. Finally, the dimension and degree are obtained by transforming the HS into the two forms of Hilbert series in theorem 2.1 and theorem 2.2. Finally, we obtain the dimension of the Hilbert series to be 40 and the degree as stated in Sec. I. With such results, we summarize them into the following theorem

Theorem 5.1: The Hilbert series for MSSM with gauge group $S U(3) \times S U(2) \times U(1)$ and particle content in Table $I$ is a rational function with its numerator being a polynomial of degree 557,392 and its denominator being in Euler form with structure shown in Eq. (5.11). The detailed results for Hilbert series can be found in this link. Particularly, the dimension obtained from the HS is 40 and the degree is shown in Sec. I, where the dimension is independent of choice of weights whereas the degree depends on the particular choices.

\section{ACKNOWLEDGMENTS}

Y.H.H. is indebted to the Science and Technology Facilities Council, UK, for Grant No. ST/J00037X/1, the Chinese Ministry of Education, for a Chang-Jiang Chair Professorship at NanKai University, and the city of Tian-Jin for a Qian-Ren Award. Y.X. is grateful for the City Doctoral Scholarship for its generous support.

\section{APPENDIX A: ILLUSTRATIVE EXAMPLES FOR THE PLETHYSTIC PROGRAM}

The first part of this Appendix reviews the application of plethystics in converting between single- and multitrace partition functions that count BPS operators.

\section{Single-Trace at $N \rightarrow \infty$}

To familiarize ourselves with the definitions in Eq. (2.11), we take $\mathbb{C}^{3}$ for illustration. This comes from the AdS/CFT correspondence where the CY threefold is simply $\mathbb{C}^{3}$ with associating Sasaki-Einstein manifold being $S^{5}$. There is no baryonic charge since the third homology of $S^{5}$ is trivial and the isometry group is $S U(4)$ of rank 3, meaning CY manifold is toric and has $3 U(1)$ charges. So we can define 3 variables $t_{1}, t_{2}, t_{3}$ for measuring charges in their powers. The $\mathcal{N}=4 U(N)$ gauge theory in $\mathcal{N}=1$ language has three adjoint chiral multiplets $x, y$ and $z$. Since we want to count GIOs, we therefore need to impose F-term relations from superpotential $W=\operatorname{Tr}(x[y, z])$. By solving these constraints, we have the relation $[x, y]=[y, z]=$ $[z, x]=0$. Therefore, a generic single-trace GIO in the chiral ring will be of the form $\operatorname{Tr}\left(x^{i} y^{j} z^{k}\right)$. Then we can assign $t_{1}$ to count the number of field $x, t_{2}$ the number of $y$ and $t_{3}$, the number of $z$. Putting together, we have the generating function to be

$$
\begin{aligned}
f\left(t_{1}, t_{2}, t_{3} ; \mathbb{C}^{3}\right) & =\sum_{i=0}^{\infty} \sum_{j=0}^{\infty} \sum_{k=0}^{\infty} t_{1}^{i} t_{2}^{j} t_{3}^{k} \\
& =\frac{1}{\left(1-t_{1}\right)\left(1-t_{2}\right)\left(1-t_{3}\right)}
\end{aligned}
$$

This result becomes exact when we take the limit $N \rightarrow \infty$. In the next paragraph, we direct out attention to the relation between single- and multitrace generating function via plethystic exponential.

\section{Multi-Trace at $N \rightarrow \infty$}

For the case of a single D3-brane on $\mathbb{C}^{3}$, the adjoint fields $x, y$ and $z$ are simple complex numbers and thus any product of these fields are multitrace operators. Therefore, we only have 4 single trace operators: the identity, $x, y$ and $z$. So the generating function for single-trace becomes

$$
f_{1}\left(t_{1}, t_{2}, t_{3}\right)=1+t_{1}+t_{2}+t_{3} .
$$

Now we look at the single-trace generating function for $N \rightarrow \infty$, which is Eq. (A1). Each of such operators is represented by a monomial $t_{1}^{i} t_{2}^{j} t_{3}^{k}$, which can be interpreted as a multitrace operator for just $N=1$ or one D3-brane. Therefore, this means $g_{1}$, the generating function for multitrace operators on a single D3-brane is the same as $f_{\infty}$, the generating function for single-trace operators for infinite D3 branes: $g_{1}=f_{\infty}$. Now we find the relation between $f_{1}$ and $g_{1}$ : 


$$
\begin{aligned}
g_{1}\left(t_{1}, t_{2}, t_{3}\right) & =\frac{1}{\left(1-t_{1}\right)\left(1-t_{2}\right)\left(1-t_{3}\right)} \\
& =\exp \left[-\log \left(1-t_{1}\right)-\log \left(1-t_{2}\right)-\log \left(1-t_{3}\right)\right] \\
& =\exp \left(\sum_{r=1}^{\infty} \frac{t_{1}^{r}+t_{2}^{r}+t_{3}^{r}}{r}\right) \\
& =\exp \left(\sum_{r=1}^{\infty} \frac{f_{1}\left(t_{1}^{r}, t_{2}^{r}, t_{3}^{r}\right)-1}{r}\right) .
\end{aligned}
$$

We can see from the above that the function $g_{1}$ is the plethystic exponential of $f_{1}$ and this relation in fact generalize to any value of $N$.

After this short review on plethystic exponential, we see that it is a combinatoric tool for generating the Hilbert series or simply a generating function of all symmetric combination of its argument. It is interesting to see that the inverse of the exponential also contains certain geometric information as we shall shortly cover. The definition of plethystic logarithm is as follows:

$$
f(t)=P E^{-1}(g(t))=\sum_{k=1}^{\infty} \frac{\mu(k)}{k} \log \left(g\left(t^{k}\right)\right),
$$

where $\mu(k)$ is the Möbius function

$\mu(k)= \begin{cases}0 & k \text { has one or more repeated prime factors, } \\ 1 & k=1, \\ (-1)^{n} & k \text { is a product of } n \text { distinct primes. }\end{cases}$

To illustrate the reverse of the plethystic exponential, i.e the plethystic logarithm, we use two examples: (1) the simplest non-Abelian subgroup of $S U$ (3) Valentiner group, $\Delta\left(3 \cdot 3^{2}\right)$ and (2) the simple Abelian $\mathbb{Z}_{3}$, to illustrate how we can obtain the information on the generators and syzygies thereof for $\mathbb{C}^{3} / \Delta\left(3 \cdot 3^{2}\right)$ and $\mathbb{C}^{3} / \mathbb{Z}^{3}$ as we as the determination of whether these two orbifolds are complete intersections. Consider the simplest nonAbelian discrete subgroup of $S U(3)$, i.e., the Valentiner group $\Delta\left(3 \cdot 3^{2}\right)$, defined as

$\Delta(27):=\left\langle\left(\begin{array}{ccc}\omega_{3} & 0 & 0 \\ 0 & 1 & 0 \\ 0 & 0 & \omega_{3}^{-1}\end{array}\right),\left(\begin{array}{ccc}1 & 0 & 0 \\ 0 & \omega_{3} & 0 \\ 0 & 0 & \omega_{3}^{-1}\end{array}\right),\left(\begin{array}{lll}0 & 1 & 0 \\ 0 & 0 & 1 \\ 1 & 0 & 0\end{array}\right)\right\rangle$.

The Molien series is given by

$$
\begin{aligned}
M(t ; \Delta(27))= & \frac{-1+t^{3}-t^{6}}{\left(-1+t^{3}\right)^{3}} \\
= & 1+2 t^{3}+4 t^{6}+7 t^{9}+11 t^{12}+16 t^{15} \\
& +22 t^{18}+\cdots
\end{aligned}
$$

First we need to construct its invariant generators and syzygies using a technique from Reynolds and Gröbner basis. Then we can check these results against those from plethystic logarithm.

The defining equations (syzygies), are constrained by the order of the group $\Delta\left(3 \cdot 3^{2}\right)^{6}$ and we can construct this finite set of invariants. There is an averaging technique due to O. Reynolds (c.f. [53]. Given some polynomial $F(x)$, one can construct the Reynolds operator

$$
R_{G}[F(x)]:=\frac{1}{|g|} \sum_{g \in G} F(g \circ x) .
$$

Then the polynomial $R_{G}[F(x)]$ is invariant under $G$ by construction. Therefore, we go up to degree 27 to find the list of invariants for group $\Delta\left(3 \cdot 3^{2}\right)$. More specifically, there are 174 invariants of degrees $0,3,6, \ldots, 24,27$. With Gröbner basis, we find that there are only 4 nontrivial generators for there 174 polynomials:

$$
\begin{aligned}
\{m & =2 x y z, n=x^{3}+y^{3}+z^{3}, p=x^{6}+y^{6}+z^{6}, \\
q & \left.=x^{3} y^{6}+x^{6} z^{3}+y^{3} z^{6}\right\} .
\end{aligned}
$$

We also find a single relation in $\mathbb{C}[m, n, p, q]$ :

$$
\begin{aligned}
8 m^{6} & +m^{3}\left(-48 n^{3}+72 n p+72 q\right) \\
& +81\left(\left(n^{2}-p\right)^{3}-4 n\left(n^{2}-p\right) q+8 q^{2}\right)=0 .
\end{aligned}
$$

So we find that $\mathbb{C}^{3} / \Delta\left(3 \cdot 3^{2}\right)$ is a complete intersection given by a single hypersurface in $\mathbb{C}^{4}$.

Let us turn to plethystic logarithm, we find it for $\Delta\left(3 \cdot 3^{2}\right)$ to be

$$
f_{1}=P E^{-1}\left(\frac{-1+t^{3}-t^{6}}{\left(-1+t^{3}\right)^{3}}\right)=2 t^{3}+t^{6}+t^{9}-t^{18} .
$$

We see that the RHS terminates and it can be interpreted as follows: there are 2 degree 3 invariants, 1 degree 6 and 1 degree 9 invariant, these 4 invariants obey a single relation of total degree 18. Comparing this with Eq. (A6), we indeed see that this is the defining relation for $\mathbb{C}^{3} / \Delta\left(3 \cdot 3^{2}\right)$. In fact, the finiteness of plethystic logarithm indicates that the underlying variety is a complete intersection, i.e., the number of defining equation is equal to the codimension of the variety in the embedding space. The story for noncomplete intersection has more content to it. Now let us look at the Abelian orbifold $\mathbb{C}^{3} / \mathbb{Z}^{3}$, which is toric and also $d P_{0}$ as a cone over $\mathbb{P}^{2}$. For the group action $(x, y, z) \rightarrow \omega_{3}(x, y, z)$, we can construct the Molien series to be

\footnotetext{
${ }^{6}$ Specifically, this is a theorem due to Nöther: The polynomial ring of invariants is finitely generated and the degree of the generators is bounded by the order of the group $|G|$.
} 


$$
f_{\infty}(t)=M\left(t, \mathbb{Z}_{3}\right)=\frac{1+7 t^{3}+t^{6}}{\left(1-t^{3}\right)^{3}}
$$

where we can get the plethystic logarithm to be

$$
\begin{aligned}
f_{1}(t) & =P E^{-1}\left[f_{\infty}(t)\right] \\
& =10 t^{3}-27 t^{6}+105 t^{9}-540 t^{12}+3024 t^{15}+\mathcal{O}\left(t^{18}\right) .
\end{aligned}
$$

This agrees with known facts that the equation for this orbifold is 27 quadrics in $\mathbb{C}^{10}$, that is 10 degree three invariants satisfying 27 relations of degree 6 . However, these information are only included in the first two terms in the series and the rest of the terms are a reflection of the fact that we no longer have a complete intersection. Therefore, the plethystic logarithm of the Hilbert series is no longer a polynomial and continues ad infinitum. In this final paragraph, let us explain why the plethystic logarithms for noncomplete intersections are infinite. First, the Poincaré series is always a rational function when simplified and collected. Particularly, the denominator of the series is of the form of products of $\left(1-t^{k}\right)$ with possible repeats of $k$ while the numerator being some complicated polynomial. We call this the Euler form. When taking plethystic logarithm, we are essentially trying to solve the following problem: find integers $b_{n}$ such that

$$
f(t)=\frac{1}{\prod_{n=1}^{\infty}\left(1-t^{n}\right)^{b_{n}}},
$$

where $f(t)$ is a rational function in Euler form. Note that $P E^{-1}[f(t)]=\sum_{n=1}^{\infty} b_{n} t^{n}$ does not need to have all positive $b_{n}$. Since the denominator is already in form of products of $\left(1-t^{n}\right)$, positive values of $n$ and $b_{n}$ can be read off immediately. The numerator in the rational function gives the negative values of $b_{n}$ and contribute to the relations among invariants. For example, we can find $b_{n}$ for $\Delta\left(3 \cdot 3^{2}\right)$

$$
\frac{1-t^{3}+t^{6}}{\left(1-t^{3}\right)^{3}}=\frac{\left(1-t^{18}\right)}{\left(1-t^{6}\right)\left(1-t^{9}\right)\left(1-t^{3}\right)^{2}}=\frac{1}{\prod_{n=1}^{\infty}\left(1-t^{n}\right)^{b_{n}}},
$$

where we used the identity

$$
\frac{\left(1-t^{3}\right)\left(1-t^{18}\right)}{\left(1-t^{6}\right)\left(1-t^{9}\right)}=1-t^{3}+t^{6}
$$

Now we find the solution: the denominator contributes terms $2 t^{3}, t^{6}$ and $t^{9}$ and the numerator contributes the terms $-t^{18}$. Thus, $P E^{-1}[M(t)]=2 t^{3}+t^{6}+t^{9}-t^{18}$. This means there are 2 degree 3,1 degree 6 , and 1 degree 9 invariants, obeying a single degree 18 relation. The crucial fact that the numerator can be factorized into Euler form dictates that the plethystic logarithm terminates in a series expansion. Therefore, finding relation in this language corresponds to finding factorizations of the numerator into Euler form. Take $\mathbb{C}^{3} / \mathbb{Z}_{3}$, we have its Poincaré series as $\left(1-7 t^{3}+t^{6}\right) /\left(1-t^{3}\right)^{3}$. No rational identity can put the numerator $1-7 t^{3}+t^{6}$ into Euler form and the plethystic logarithm does not terminate. If we convert the numerator into Euler form, we get

$$
1+7 t^{3}+t^{6}=\frac{\left(1-t^{6}\right)^{27}\left(1-t^{12}\right)^{540} \cdots}{\left(1-t^{3}\right)^{7}\left(1-t^{9}\right)^{105} \cdots}
$$

where we have $10 t^{3}$ from $\left(1-t^{3}\right)^{10}$ and $-27 t^{6}$ from $\left(1-t^{6}\right)^{27}$. However, for higher degree invariants, i.e., 28 degree 6 and 55 degree 9 invariants, etc., we need further expansion on both top and bottom for Eq. (A9). Using computer package such as Mccaulay2, we can find 595 relations for 10 degree 3 and 28 degree 6 invariants: 55 of degree 6, 225 of degree 9 and 315 of degree 12. This thus reads

$$
\begin{gathered}
10 t^{3}+28 t^{6}-55 t^{6}-225 t^{9}-315 t^{12} \\
=10 t^{3}-27 t^{6}-225 t^{9}-315 t^{12} .
\end{gathered}
$$

For higher degree invariants and relations, we can correct the coefficients for higher order terms such as $t^{9}$ and $t^{12}$.

\section{APPENDIX B: EXTENDED SYNTHETIC DIVISION}

In this section, we review some basic materials of extended synthetic division with the Python implementation codes presented. Synthetic division is a method of performing Euclidean division of polynomials with less calculation than regular polynomial long division. It is first developed for division by monomial of the form $x-a$, but later generalized to division by any monomials and polynomials. The advantage of this method is that it allows one to calculate division without writing out variables and it uses less calculations. Let us first look at a simple example:

$$
\frac{x^{3}-12 x^{2}-42}{x^{2}+x-3}
$$

The steps are as follows

(1) We negate them as before and write every coefficients but the first on to the left of the bar in an upward.

(2) We copy the first coefficient and multiply the diagonal by the copied number and place them diagonally to the right from the copied entry.

(3) We sum up the next column until we go past the entries at the top with the next diagonal multiplication.

(4) We sum up the remaining column. Since there are two entries to the left of the bar, so the remainder is of degree 1 . We then mark the separation with a vertical bar as 


$$
1 x-13 \mid 16 x-81,
$$

so we have the final quotient and remainder as

$$
\frac{x^{3}-12 x^{2}-42}{x^{2}+x-3}=x-13+\frac{16 x-81}{x^{2}+x-3}
$$

We present the division here for the convenience of the reader

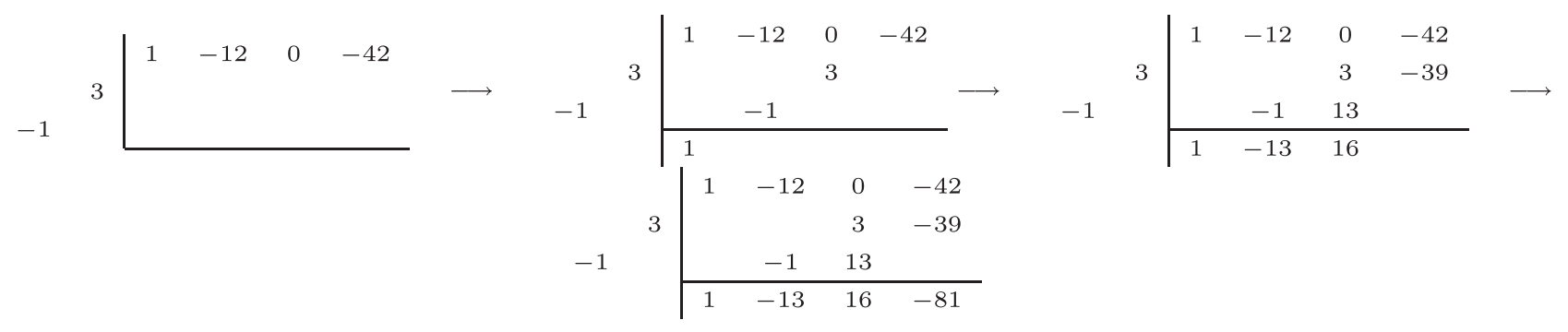

More specifically, we present a Python implementation of the algorithm here

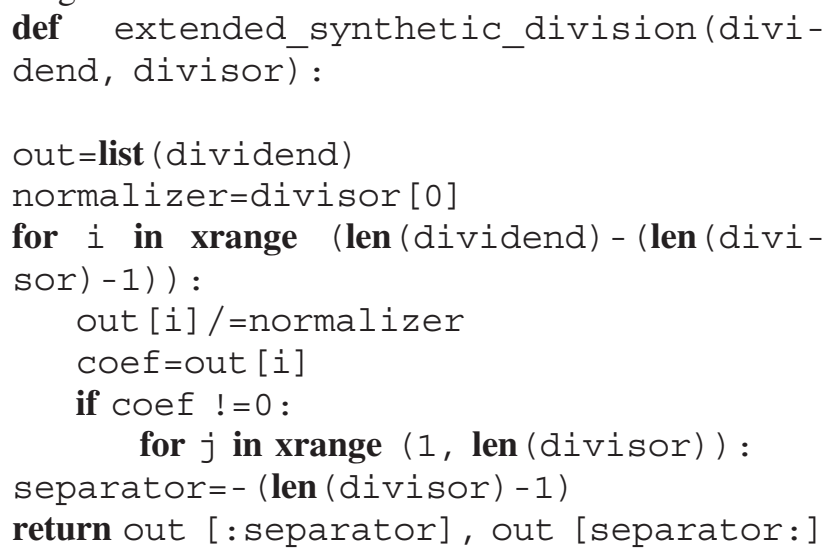

\section{APPENDIX C: HILBERT SERIES GRBNER BASIS AND EXAMPLES FROM COMMUTATIVE ALGEBRA}

In this Appendix, we review some of the foundation of Hilbert series and see how it is constructed for specific counting purposes. First, we are most interested in polynomial ring $K\left[x_{1}, \ldots, x_{n}\right]$ consisting polynomials in variables $x_{1}, \ldots, x_{n}$ with coefficients in the ring $K$. We typically take $K$ to be a field, such as real numbers $\mathbb{R}$. We also have monomials in the form $x_{1}^{\alpha_{1}} \cdots x_{n}^{\alpha_{n}}$, whose linear combination gives a polynomials. So monomials serve as building blocks for polynomials via addition. Since we are ultimately interested in counting things in polynomial ring using Hilbert series, we would like to simplify this counting to monomial level. Therefore, the notion of grading is introduced for this counting purpose. On the physical side story, the grading is usually from the charges of certain global symmetries. Let us look at some natural choice for grading, the degree of a polynomial, defined as $\operatorname{deg}\left(x_{1}^{\alpha_{1}} \cdots x_{n}^{\alpha_{n}}\right)=\alpha_{1}+\cdots+\alpha_{n}$. Adding up monomials of the same degree gives us a homogeneous polynomial. Using this notation, we can decompose a set of all homogeneous, degree $k$ polynomials $R_{k}$ into direct sum $R=\bigoplus_{k \in \mathbb{N}} R_{k}$. Mathematically, variables $x_{1}, \ldots, x_{n}$ are said to form a $\mathbb{N}$ graded algebra.

The dimension of $R_{k}$ is defined to be the number of independent degree $k$ monomials. A Hilbert function is defined as $H F(R, k)=\operatorname{dim}\left(R_{k}\right)$. The Hilbert series is then naturally defined as

$$
H(R, t)=\sum_{k} H F(R, k) t^{k} .
$$

For polynomial ring $R=K\left[x_{1}, \ldots, x_{n}\right]$, to construct a degree $k$ monomial, we need to choose $k$ items from $n$ candidates, with multiples being allowed, i.e.,

$H F\left(K\left[x_{1}, \ldots, x_{n}\right], k\right)=\sum_{k_{1}+k_{2}+\cdots+k_{n}=k}\left(\begin{array}{c}n+k-1 \\ k\end{array}\right)$,

and the Hilbert series is

$H\left(K\left[x_{1}, \ldots, x_{n}\right], k\right)=\sum_{k=0}^{\infty}\left(\begin{array}{c}n+k-1 \\ k\end{array}\right) t^{k}=\frac{1}{(1-t)^{n}}$.

The power of the denominator actually shows that there are $n$ degree 1 generators with no relations among them. Note that this identity also gives the generating function of multiset coefficients. 
Now let us discuss a bit more about ideal in a polynomial ring. First take $s$ polynomials from the ring, $f_{1}$, $f_{2}, \ldots, f_{s} \in K\left[x_{1}, \ldots, x_{n}\right]$. Then the variety $V$ defined by $f_{i}$ are the points in $K^{n}$, which are zeroes of the $s$ polynomials. The ideal $\left\langle f_{1}, \ldots, f_{s}\right\rangle$ is then the set of polynomials that vanishes on $V$. With this definition in hand, we can proceed to define quotient variety now. Let $R=K\left[x_{1}, \ldots, x_{n}\right]$ be a polynomial ring graded by degree and let $I=\left\langle f_{1}, \ldots, f_{s}\right\rangle$ be an ideal of $R$. Formally, the ideal formed by polynomials $f_{1}, \ldots f_{s} \in K\left[x_{1}, \ldots, x_{n}\right]$ is the set of polynomials obtained by taking $f_{i}$ as basis vectors, with coefficients $h_{i}$ from $K\left[x_{1}, \ldots x_{n}\right]$

$$
\left\langle f_{1}, \ldots, f_{s}\right\rangle=\left\{\sum_{i=1}^{s} h_{i} f_{i}: h_{1}, \ldots, h_{s} \in K\left[x_{1}, \ldots, x_{n}\right]\right\} .
$$

We can quotient the ring by the ideal,

$$
M=R / I \text {. }
$$

So by definition, $M$ is made of equivalence classes of polynomials. So in this sense, the elements of ideal are zero polynomials and are removed by this quotient procedure, where the algebraic structure is preserve as $M$ is also a ring. For homogenous ideal, the quotient ring derived from it is then defined to be a graded module since the grading is understood to be the degree of polynomials. This means the quotient preserves the grading and $M$ is decomposed as a direct sum $M=\oplus_{k} M_{k}$, where $M_{k}$ is the set of homogenous polynomials. So the Hilbert function for $M$ is then defined to be

$$
H F(M, k)=\operatorname{dim}\left(M_{k}\right)=\operatorname{dim}\left(R_{k}\right)-\operatorname{dim}\left(I_{k}\right) .
$$

The Hilbert series is then $H(M, t)=\sum_{k} H F(M, k) t^{k}$.

Usually, we would like to construct the quotient ring $M$ by finding a typical ideal, which is usually generated by a few polynomials $f_{1}, \ldots, f_{s}$. Then questions such as inclusion of a polynomial inside the ideal and nontrivial relations among generators, arise in this process. The answers to these questions are computational and generally quite hard. However, a special set of basis of the ideal, called Gröbner basis can be constructed most efficiently to describe the polynomial sequence $f_{1}, \ldots, f_{s}$. Now we denote the set of polynomials in Gröbner basis by $g_{1}, \ldots, g_{r}$, where $r \neq s$ in general. Since $f_{i}$ are taken as basis vectors for the ideal, we can change the basis to the new Gröbner basis, which simply generate the same ideal. A more thorough treatment for this topic can be found in [43]. To construct the Gröbner basis, we need to define an ordering of monomial first. ${ }^{7}$

\footnotetext{
${ }^{7}$ The common choices of monomial ordering are lexographic, graded lexographic and graded reverse lexographic ordering. Take two monomials $\mathbf{x}^{\alpha_{1}} \ldots x_{n}^{\alpha_{n}}$ and $\mathbf{x}^{\beta_{1}} \ldots x_{n}^{\beta_{n}}$ of total degree $\alpha=$ $\alpha_{1}+\cdots+\alpha_{n}$ and $\beta=\beta_{1}+\cdots+\beta_{n}$. We take $\mathbf{x}^{\alpha}>\mathbf{x}^{\beta}$ if $\alpha>\beta$; if $\alpha=\beta$, then $\mathbf{x}^{\alpha}>\mathbf{x}^{\beta}$ if $\alpha_{1}>\beta_{1}$; if $\alpha=\beta$ and $\alpha_{1}=\beta_{1}$, then $\mathbf{x}^{\alpha}>\mathbf{x}^{\beta}$ if $\alpha_{2}>\beta_{2}$ and so on.
}

This monomial ordering " $>$ " determines whether $\mathbf{x}^{\alpha}>\mathbf{x}^{\beta}, \mathbf{x}^{\alpha}=\mathbf{x}^{\beta}$ or $\mathbf{x}^{\alpha}<\mathbf{x}^{\beta}$ for two monomials $\mathbf{x}^{\alpha}=$ $x_{1}^{\alpha_{1}} \cdots x_{n}^{\alpha_{n}}$ and $\mathbf{x}^{\beta}=x_{1}^{\beta_{1}} \cdots x_{n}^{\beta_{n}}$. With this ordering, we can then find the "largest" monomial inside a polynomial $h \in K\left[x_{1}, \ldots, x_{n}\right]$. This is defined to be the initial monomial of $h$, denoted by $\operatorname{in}(h) .{ }^{8}$ For every polynomial in $I=\left\langle f_{1}, \ldots, f_{s}\right\rangle$, we take their initial monomial and denote this set to be in $(I)$. In general, in $(I)$ is not equal to the set generated by initial monomials of the $f_{i}$. But the defining property of Gröbner basis is that $\operatorname{in}(I)=\left\langle\operatorname{in}\left(g_{1}\right), \ldots, \operatorname{in}\left(g_{r}\right)\right\rangle$.

With the above abstract definition, we shall benefit from a few concrete examples. First let us take the polynomial ring of two variables with coefficients in the real numbers, $R=\mathbb{R}[x, y]$. Here we take monomial ordering to be graded reverse lexographic ordering, which is the default setting for computer package Mccaulay2.

Example 1: Let $R=\mathbb{R}] x, y]$ and $I=\langle x+y\rangle$. As the ideal has just a single polynomial, it is then by definition, a Gröbner basis. Hence, the initial ideal is generated by the $\operatorname{in}(x+y)=x$, in $(I)=\langle x\rangle$. The Hilbert series for the quotient ring $M=R / I$ is equivalent to the Hilbert series of $R / \operatorname{in}(I)=\mathbb{R}[x, y] /\langle x\rangle=\mathbb{R}[y]$. Therefore, we have

$$
H(\mathbb{R}[x, y] /\langle x+y\rangle, t)=\frac{1}{1-t} .
$$

Example 2: Let $R=\mathbb{R}[x, y]$ and $I=\left\langle x^{2}, y^{3}\right\rangle$. A monomial of the form $x^{\alpha} y^{\beta}$ is in the ideal for $\alpha \geq 2$ and $\beta \geq 3$. Therefore, the monomials for the quotient ring are $1, x, y, x y, y^{2}, x y^{2}$. Since the Hilbert series counts the independent monomials, it is then

$$
H\left(\mathbb{R}[x, y] /\left\langle x^{2}, y^{3}\right\rangle, t\right)=1+2 t+2 t^{2}+t^{3} .
$$

This finite polynomial hints us that it can be written as a rational function with both numerator and denominator being in Euler form. This is actually

$$
H=\frac{\left(1-t^{2}\right)\left(1-t^{3}\right)}{(1-t)^{2}}=\frac{1-t^{2}-t^{3}+t^{5}}{(1-t)^{2}}
$$

where the denominator is the Hilbert series of free ring $\mathbb{R}[x, y]$, while the numerator reflects the relation among generators of the ideal.

\footnotetext{
${ }^{8}$ This is also commonly defined as the leading term of $h$ and denoted by $\operatorname{LT}(h)$.
} 
[1] F. Buccella, J. P. Derendinger, S. Ferrara, and C. A. Savoy, Patterns of symmetry breaking in supersymmetric gauge theories, Phys. Lett. 115B, 375 (1982).

[2] R. Gatto and G. Sartori, Consequences of the complex character of the internal symmetry in supersymmetric theories, Commun. Math. Phys. 109, 327 (1987).

[3] C. Procesi and G. W. Schwarz, The geometry of orbit spaces and gauge symmetry breaking in supersymmetric gauge theories, Phys. Lett. 161B, 117 (1985).

[4] M. A. Luty and W. I. Taylor, Varieties of vacua in classical supersymmetric gauge theories, Phys. Rev. D 53, 3399 (1996).

[5] T. Gherghetta, C. F. Kolda, and S. P. Martin, Flat directions in the scalar potential of the supersymmetric standard model, Nucl. Phys. B468, 37 (1996).

[6] J. Gray, Y. H. He, V. Jejjala, and B. D. Nelson, Vacuum geometry and the search for new physics, Phys. Lett. B 638, 253 (2006).

[7] J. Gray, Y. H. He, V. Jejjala, and B. D. Nelson, Exploring the vacuum geometry of $N=1$ gauge theories, Nucl. Phys. B750, 1 (2006).

[8] J. Gray, A. Hanany, Y. H. He, V. Jejjala, and N. Mekareeya, SQCD: A geometric apercu, J. High Energy Phys. 05 (2008) 099.

[9] Y. H. He, V. Jejjala, C. Matti, and B. D. Nelson, Veronese geometry and the electroweak vacuum moduli space, Phys. Lett. B 736, 20 (2014).

[10] Y. H. He, V. Jejjala, C. Matti, B. D. Nelson, and M. Stillman, The geometry of generations, Commun. Math. Phys. 339, 149 (2015).

[11] Y. H. He, V. Jejjala, C. Matti, and B. D. Nelson, Testing Rparity with geometry, J. High Energy Phys. 03 (2016) 079.

[12] S. Benvenuti, B. Feng, A. Hanany, and Y. H. He, Counting BPS operators in gauge theories: Quivers, syzygies and plethystics, J. High Energy Phys. 11 (2007) 050.

[13] B. Feng, A. Hanany, and Y.H. He, Counting gauge invariants: The plethystic program, J. High Energy Phys. 03 (2007) 090.

[14] B. Feng, A. Hanany, and Y. H. He, D-brane gauge theories from toric singularities and toric duality, Nucl. Phys. B595, 165 (2001).

[15] J. M. Maldacena, The large N limit of superconformal field theories and supergravity, Int. J. Theor. Phys. 38, 1113 (1999); Adv. Theor. Math. Phys. 2, 231 (1998).

[16] P. Pouliot, Molien function for duality, J. High Energy Phys. 01 (1999) 021.

[17] C. Romelsberger, Counting chiral primaries in $N=1, d=4$ superconformal field theories, Nucl. Phys. B747, 329 (2006).

[18] C. Romelsberger, Calculating the superconformal index and seiberg duality, arXiv:0707.3702.

[19] F. A. Dolan, Counting BPS operators in $N=4$ SYM, Nucl. Phys. B790, 432 (2008).

[20] F. A. Dolan and H. Osborn, Applications of the superconformal index for protected operators and q-hypergeometric identities to $N=1$ dual theories, Nucl. Phys. B818, 137 (2009).

[21] A. Hanany and C. Romelsberger, Counting BPS operators in the chiral ring of $N=2$ supersymmetric gauge theories or
$N=2$ braine surgery, Adv. Theor. Math. Phys. 11, 1091 (2007).

[22] B. Sundborg, The Hagedorn transition, deconfinement and $N=4$ SYM theory, Nucl. Phys. B573, 349 (2000).

[23] T. W. Brown, P. J. Heslop, and S. Ramgoolam, Diagonal multi-matrix correlators and BPS operators in $N=4 \mathrm{SYM}$, J. High Energy Phys. 02 (2008) 030.

[24] A. Hanany, N. Mekareeya, and G. Torri, The Hilbert series of adjoint SQCD, Nucl. Phys. B825, 52 (2010).

[25] A. Hanany and N. Mekareeya, Counting gauge invariant operators in SQCD with classical gauge groups, J. High Energy Phys. 10 (2008) 012.

[26] D. Forcella, A. Hanany, and A. Zaffaroni, Baryonic generating functions, J. High Energy Phys. 12 (2007) 022.

[27] A. Hanany, Counting BPS operators in the chiral ring: The plethystic story, AIP Conf. Proc. 939, 165 (2007).

[28] V. Balasubramanian, B. Czech, Y. H. He, K. Larjo, and J. Simon, Typicality, black hole microstates and superconformal field theories, J. High Energy Phys. 03 (2008) 008.

[29] A. Butti, D. Forcella, A. Hanany, D. Vegh, and A. Zaffaroni, Counting chiral operators in quiver gauge theories, J. High Energy Phys. 11 (2007) 092.

[30] D. Forcella, A. Hanany, Y. H. He, and A. Zaffaroni, The master space of $N=1$ gauge theories, J. High Energy Phys. 08 (2008) 012; D. Forcella, A. Hanany, Y. H. He, and A. Zaffaroni, Mastering the master space, Lett. Math. Phys. 85, 163 (2008).

[31] S. Cremonesi, 3d supersymmetric gauge theories and Hilbert series, Proc. Symp. Pure Math. 98, 21 (2018).

[32] S. Cremonesi, N. Mekareeya, and A. Zaffaroni, The moduli spaces of $3 \mathrm{~d} \mathcal{N} \geq 2$ Chern-Simons gauge theories and their Hilbert series, J. High Energy Phys. 10 (2016) 046.

[33] A. Hanany and R. K. Seong, Hilbert series and moduli spaces of $k \mathrm{U}(\mathrm{N})$ vortices, J. High Energy Phys. 02 (2015) 012.

[34] A. Dey, A. Hanany, N. Mekareeya, D. Rodrguez-Gómez, and R.K. Seong, Hilbert series for moduli spaces of instantons on $C^{2} / Z_{n}$, J. High Energy Phys. 01 (2014) 182.

[35] D. Rodrguez-Gmez and G. Zafrir, On the 5d instanton index as a Hilbert series, Nucl. Phys. B878, 1 (2014).

[36] N. Jokela, M. Jarvinen, and E. Keski-Vakkuri, New results for the SQCD Hilbert series, J. High Energy Phys. 03 (2012) 048.

[37] A. Hanany, E. E. Jenkins, A. V. Manohar, and G. Torri, Hilbert series for flavor invariants of the Standard Model, J. High Energy Phys. 03 (2011) 096.

[38] L. Lehman and A. Martin, Hilbert series for constructing Lagrangians: Expanding the phenomenologist's toolbox, Phys. Rev. D 91, 105014 (2015).

[39] L. Lehman and A. Martin, Low-derivative operators of the Standard Model effective field theory via Hilbert series methods, J. High Energy Phys. 02 (2016) 081.

[40] B. Henning, X. Lu, T. Melia, and H. Murayama, Hilbert series and operator bases with derivatives in effective field theories, Commun. Math. Phys. 347, 363 (2016).

[41] W. Fulton and J. Harris, Representation Theory: A First Course (Springer, New York, 1991).

[42] Y. H. He, Calabi-Yau Varieties: From Quiver representations to Dessins d'Enfants, arXiv:1611.09398. 
[43] D. Cox, J. Little, and D. O'Shea, Ideals, Varieties, and Algorithms: An Introduction to Computational Algebraic Geometry and Commutative Algebra (Springer, New York, 1992).

[44] H. Schenck, Computational Algebraic Geometry (Cambridge University Press, Cambridge, England, 2003), Vol. 58.

[45] A. Futaki, H. Ono, and Y. Sano, Hilbert series and obstructions to asymptotic semistability, Adv. Math. 226, 254 (2011).

[46] D. Martelli, J. Sparks, and S. T. Yau, Sasaki-Einstein manifolds and volume minimisation, Commun. Math. Phys. 280, 611 (2008).

[47] J. P. Gauntlett, D. Martelli, J. Sparks, and S. T. Yau, Obstructions to the existence of Sasaki-Einstein metrics, Commun. Math. Phys. 273, 803 (2007).
[48] Y. H. He, R. K. Seong, and S. T. Yau, Calabi-Yau volumes and reflexive polytopes, Commun. Math. Phys. 361, 155 (2018).

[49] T. Molien, Über die Invarianten der linearen Substitutionsgruppen. Sitzungber. Konig. Preuss. Akad. Wiss., J. Berl. Ber. 52, 1152 (1897).

[50] H. Derksen and G. Kemper, Computational Invariant Theory (Springer, New York, 2002).

[51] https://github.com/xiao-yan/MSSM_Plethystics.

[52] L. Fan, A Generalization of synthetic division and a general theorem of division of polynomials, Math. Medley 30, 30 (2003), MathWorld http://mathworld.wolfram.com/ SyntheticDivision.html.

[53] S.-T Yau and Y. Yu, Gorenstein Quotient Singularities in Dimension Three (American Mathematical Society, Providence, 1993). 